ARTICLE

\title{
Nanoscale rules governing the organization of glutamate receptors in spine synapses are subunit specific
}

\author{
Martin Hruska (1,3, Rachel E. Cain ${ }^{2,3}$ \& Matthew B. Dalva (iD ${ }^{2 凶}$
}

Heterotetrameric glutamate receptors are essential for the development, function, and plasticity of spine synapses but how they are organized to achieve this is not known. Here we show that the nanoscale organization of glutamate receptors containing specific subunits define distinct subsynaptic features. Glutamate receptors containing GluA2 or GluN1 subunits establish nanomodular elements precisely positioned relative to Synaptotagmin-1 positive presynaptic release sites that scale with spine size. Glutamate receptors containing GluA1 or GluN2B specify features that exhibit flexibility: GluA1-subunit containing AMPARs are found in larger spines, while GluN2B-subunit containing NMDARs are enriched in the smallest spines with neither following a strict modular organization. Given that the precise positioning of distinct classes of glutamate receptors is linked to diverse events including cell death and synaptic plasticity, this unexpectedly robust synaptic nanoarchitecture provides a resilient system, where nanopositioned glutamate receptor heterotetramers define specific subsynaptic regions of individual spine synapses.

\footnotetext{
${ }^{1}$ Department of Neuroscience, Rockefeller Neuroscience Institute, West Virginia University, 108 Biomedical Road, Morgantown, WV 26506, USA.

${ }^{2}$ Department of Neuroscience and Jefferson Synaptic Biology Center, Sidney Kimmel Medical College at Thomas Jefferson University, 233 South 10 th Street, Bluemle Life Sciences Building, Room 324, Philadelphia, PA 19107, USA. ${ }^{3}$ These authors contributed equally: Martin Hruska, Rachel E. Cain.

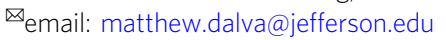


A ll glutamate receptors are heterotetrametric, composed of two obligate subunits (GluA2 or GluN1) and two subunits that help to specify function: GluN2A or GluN2B (NMDARs), or GluA1 or GluA3 (AMPARs) ${ }^{1-3}$. The heterotetrameric subunit composition of individual glutamate receptors tunes the channel's properties and is linked to specific aspects of synaptic function and plasticity ${ }^{4}$. For instance, GluA1-containing AMPARs are associated with the expression of synaptic plasticity, while GluN2B-containing NMDARs are associated with the induction of plasticity and are thought to be found at more immature and smaller spines ${ }^{3,5-8}$. The differences in the function of glutamate receptor heterotetramers have led to the suggestion that glutamate receptors containing specific subunits are differentially localized in spines of different $\operatorname{sizes}^{5,9-13}$. Despite the broad importance of subsynaptic location of glutamate receptor subunits for synaptic function, how glutamate receptor heterotetramers are organized at the nanoscale within spines is unclear.

Adding to the complexity of the synaptic nanoscale architecture, many AMPARs and NMDARs are located outside or distant to synaptic release sites ${ }^{3,14}$. These non-synaptic receptors are more mobile than AMPARs and NMDARs localized to PSDs ${ }^{15,16}$ and are thought to act as a dynamic pool of receptors that could serve as the source of new synaptically localized receptors $3,15,17-19$. While the importance of extrasynaptic and perisynaptic AMPARs and NMDARs for synaptic function has been long recognized, the relationship between the nanoscale organization of both synaptic and non-synaptic glutamate receptors at spine synapses remains controversial.

Synaptic function requires the juxtaposition of pre- and postsynaptic proteins as the precise localization of glutamate receptors relative to synaptic release sites is critical for normal synaptic function $^{20}$. AMPARs and NMDARs have distinct affinities for glutamate, with AMPARs binding glutamate at low affinity and NMDARs binding with higher affinity ${ }^{1,21}$. Electrophysiological and modeling evidence indicates that AMPARs are better coupled to glutamate release sites than NMDARs ${ }^{13}$. Moreover, the location of the heterotetrameric receptor appears important for function, with synaptic receptors signaling to induce events such as long-term potentiation and spine size changes, and nonsynaptic receptors' signaling leading to events such as long-term depression and cell death ${ }^{14,22-24}$. However, the nanoscale relationship between the positioning of AMPARs, NMDARs, and the machinery responsible for release remains unknown.

Here we demonstrate using STimulated Emission Depletion nanoscopy (STED) that the organization of NMDARs and AMPARs reflect the modular structure of pre- and postsynaptic scaffolding proteins, with the number of similar-sized clusters of these proteins scaling with spine size. However, within the modular structure of AMPARs and NMDARs, there is flexibility. Heterotetrameric AMPARs containing the GluA1 subunit and heterotetrameric NMDARs with the GluN2B subunit do not show modules that scale with spine size. Instead, they are preferentially localized to large and small spines, respectively, reflecting their function in synaptic plasticity and development. In addition to a highly ordered post-synaptic nanoarchitecture, the glutamate receptor subtypes are differentially yet precisely positioned relative to the fast calcium sensor SYT1, reflecting differences in how these receptors bind glutamate. Together, our data describe robust organizational principles of essential components of synaptic transmission and plasticity.

\section{Results}

AMPARs in dendritic spine synapses form nanoscale modules. AMPARs mediate the fast component of neurotransmission, and AMPAR abundance is correlated with spine size, determines synaptic strength, and mediates synaptic plasticity ${ }^{2,17}$. Therefore, we asked whether AMPAR nanoclusters in spines follow the modular organization defined by PSD-95 and presynaptic scaffolding proteins in spines ${ }^{25}$. To determine the relationship between glutamate receptors and pre- and post-synaptic proteins that form nanomodules, multi-color STED was chosen because STED enables super-resolution imaging of three colors with minimal chromatic aberration in $X Y$ or $Z$ planes with $\sim 50 \mathrm{~nm}$ resolution in XY (Supplementary Figs. 1 and 2a-c), and allows for discrimination of two different fluorophores as close as $\sim 70 \mathrm{~nm}$ apart (Supplementary Fig. 2d-j). All STED images were collected using $Z$-resolved STED and deconvolved using the same parameters to improve the signal to noise ratio (compare Fig. 1 and Supplementary Fig. 3). Deconvolution did not lead to significant changes in the distances between objects (Supplementary Fig. 2f, $i$, and j; see "Methods") and the size of the puncta examined were on average larger than the resolution limit of our system, measured as the minimal full width at half maximal (FWHM) detected (Supplementary Fig. 2a-c). Thus, STED enables the determination of the distance between different puncta at the nanoscale.

To visualize the heterotetrameric AMPA-type glutamatereceptor channels, we probed for the most commonly incorporated subunit, GluA2 26,27. EGFP labeled DIV21-25 neurons immunostained with antibodies for endogenous GluA2, PSD-95, and Bassoon were imaged with three-color Z-resolved 3D-STED (Fig. 1a and Supplementary Fig. 3). Neuronal morphology was determined in confocal mode, and the organization of synaptic proteins in GFP labeled spines was determined using three-color STED in $X Y$ and $Z$ planes (Fig. 1a and Supplementary Fig. 3), allowing for the organization of synaptic nanostructure to be related to spine size ${ }^{25}$. Consistent with previous results ${ }^{25}$, PSD-95 and Bassoon formed aligned nanomodules that scaled in number with increasing spine size (Fig. 1a-e and Supplementary Fig. 3).

In mature cortical neurons, $89 \%$ of spines had GluA2-containing AMPAR heterotetramers (Fig. $1 \mathrm{~b}$ and Supplementary Fig. 4b). In spines with synaptic GluA2-containing AMPARs, GluA2 subunits localized to $81 \%$ of PSD-95 nanomodules (Supplementary Fig. 4a, c). These nanoclusters of GluA2 subunits, adjacent to presynaptic markers and associated with postsynaptic PSD-95, are defined as synaptic nanoclusters (Fig. 1a, arrows and Supplementary Fig. 3, arrowheads). Non-synaptic GluA2 nanoclusters were also found in many spines (Fig. 1a, open arrowheads). To determine whether GluA2 synaptic nanoclusters form modular assemblies that scale with spine size, we determined the relationship between the number of GluA2 and PSD-95 nanoclusters in spines of different sizes. The number of GluA2 nanoclusters increased as a function of the number of PSD-95 clusters (Fig. 1d). Additionally, the number of synaptic GluA2 nanoclusters scaled linearly with spine size (Fig. 1e). The relationship of synaptic GluA2 nanocluster number to spine size was not significantly different from the scaling seen with PSD-95 and Bassoon nanomodules. These data indicate that synaptic AMPARs form nanoclusters in the same modular fashion as PSD-95.

The number, but not size, of PSD-95 nanomodules increases with plasticity-induced spine enlargement ${ }^{25}$. Therefore, we asked whether GluA2 nanoclusters might undergo similar changes in response to chemical LTP ${ }^{18,25,28,29}$. Spine enlargement was induced by application of glycine $(3 \mathrm{~min}, 200 \mu \mathrm{M})$ to DIV21-25 cortical neurons transfected with GFP. Neurons were imaged with confocal spinning disk or confocal Leica SP8 every $6 \mathrm{~min}$ for $3 \mathrm{~h}$ post glycine application $^{25}$. After live imaging, neurons were fixed and stained for endogenous GluA2, PSD-95, and Bassoon (Fig. 2a-d).

Glycine application results in significant increase in the size of $56 \%$ of spines (potentiated), with the remaining non-responsive spines having no lasting changes in spine size (Fig. 2b, e). 
a

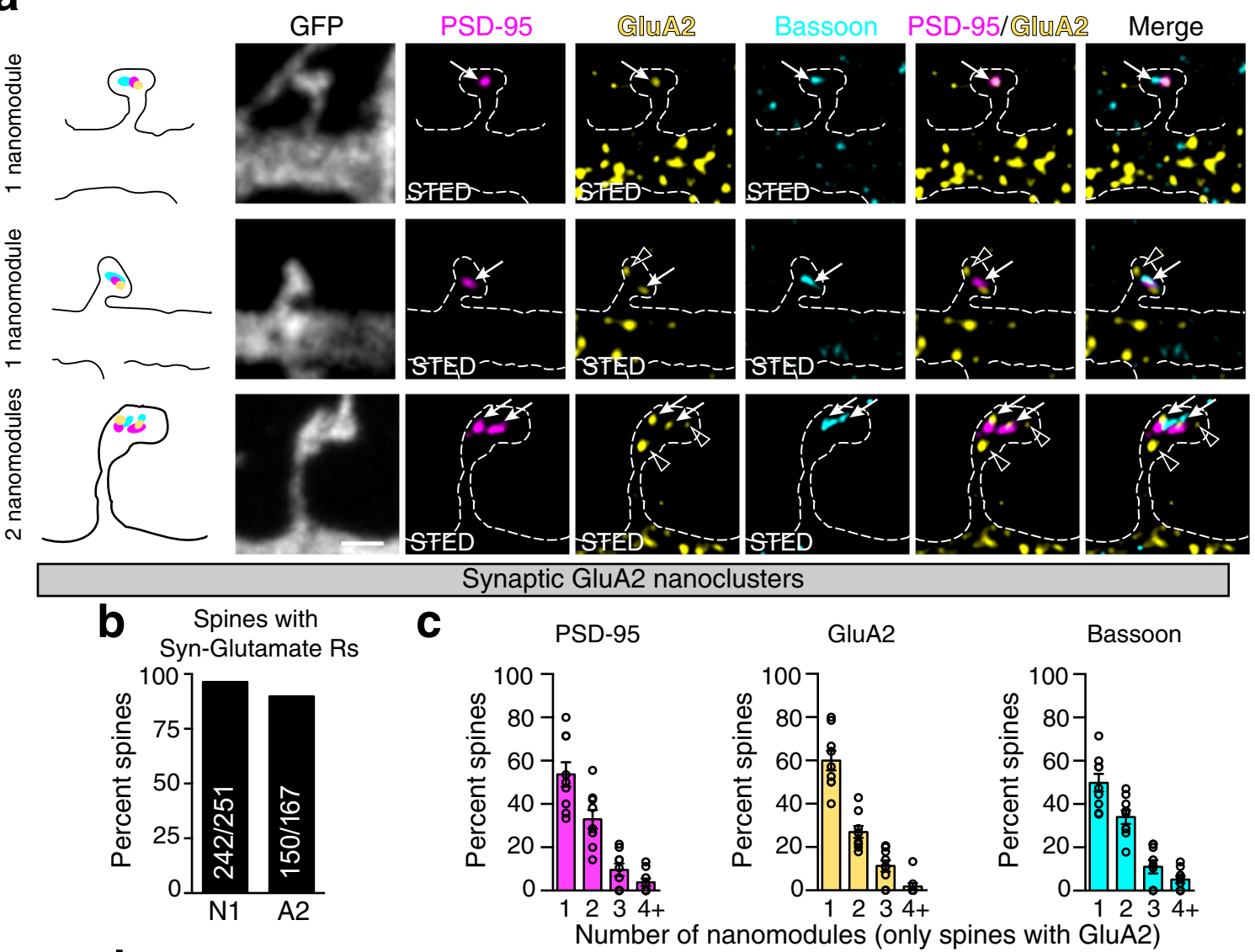

d Syn-GluA2 relationship to PSD-95 nanomodule number

e

Relationship to spine size

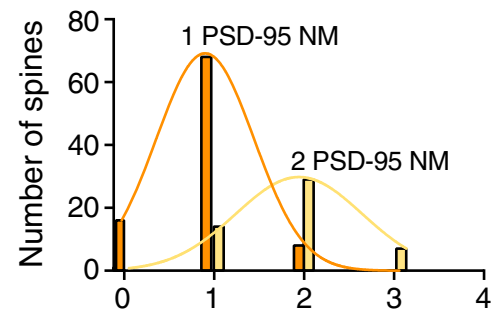

GluA2 nanomodules per spine
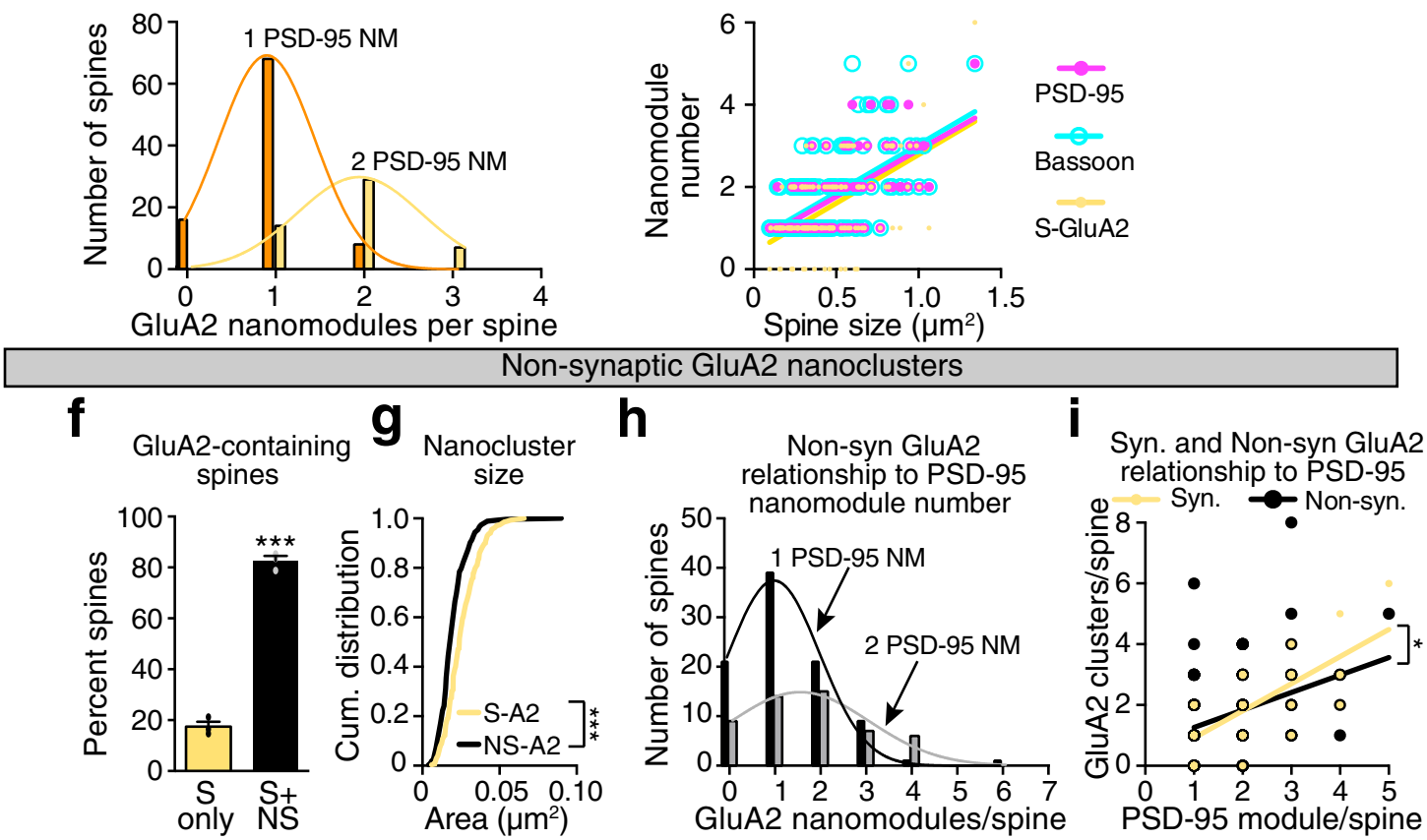

Retrospective three-color STED analysis visualized the nanoscale organization of GluA2, PSD-95, and Bassoon of individual spines that were imaged live. Potentiated spines had significantly more nanoclusters of synaptic GluA2 and PSD-95 nanomodules than control, non-responsive or MK-801/APV treated spines (Fig. 2f-h). Consistent with the model that GluA2 forms nanomodules there was no difference in the size of GluA2 nanoclusters following induction of structural plasticity (Fig. 2i). These results suggest that GluA2 forms nanomodules that scale in number with changes in spine size similar to the modular organization of other synaptic proteins ${ }^{25}$.

AMPARs dynamically associate with synapses and undergo constitutive recycling and delivery to synaptic sites to maintain synaptic strength ${ }^{17}$. Consistent with this model, the majority of 
Fig. 1 The nanoscale organization of AMPARs in dendritic spine synapses reflects a modular organization of pre- and postsynaptic adaptor molecules. a Representative images of single and two-nanomodule spines from DIV21 cortical neurons. Antibodies to PSD-95 (magenta, Atto-425), GluA2 (yellow, Atto-647N), and Bassoon (cyan, AlexaFluor-594) were used to identify the colocalization of AMPARs with synaptic adaptor nanomodules in STED mode (white arrows) in GFP-labeled spines (gray, dotted lines, confocal mode) as well as non-synaptic GluA2 clusters (open arrowheads). Scale bar: $1 \mu$ m. b Percent of spines containing synaptic NMDARs and AMPARs (numbers of spines are indicated on the graph). c Percentage of spines containing single and multiple PSD-95, GluA2, and Bassoon clusters ( $n=150$ spines). d Distribution of the number of synaptic GluA2 nanoclusters as a function of the number of PSD-95 nanomodules in a spine. Gaussian fits model distinct distributions of S-GluA2 puncta in one (orange) and two (yellow) PSD-95 nanomodule (PSD95 NM) spines ( $p<0.0001$ by extra sum of squares, F test). Most spines contain equal numbers of synaptic GluA2 and PSD-95 nanoclusters. e Quantification of the relationship between spine size ( $n=167$ spines) and the number of Bassoon (cyan line, $R^{2}=0.3537$, slope $\left.=2.337 \pm 0.2459\right)$, PSD-95 (magenta line, $R^{2}=0.4160$, slope $=2.275 \pm 0.2099$ ) and GluA2 (yellow line, $R^{2}=0.3271$, slope $=2.364 \pm 0.2639, p=0.9652$, one-way ANCOVA) clusters. $\mathbf{f}$ Percent of spines containing only synaptic (yellow bar, $n=30$ spines) or both, synaptic and non-synaptic (black bar, $n=137$ spines) GluA2 nanoclusters ( ${ }^{\star \star \star} p<0.0001$, two-tailed Student's t-test, dots represent averages of data from three individual experiments). $\mathbf{g}$ Cumulative probability distributions of synaptic and non-synaptic GluA2 nanocluster sizes ( ${ }^{\star \star \star} p<0.0001$, two-tailed Kolmogorov-Smirnov (K-S) test). $\mathbf{h}$ Distribution of the number of non-synaptic GluA2 nanoclusters relative to the number of PSD-95 nanomodules per spine as in (d). Distributions of NS-GluA2 puncta in one (black) and two (gray) PSD-95 NMs. The distributions of NS-GluA2 puncta were not related to synaptic PSD-95 puncta number ( $p=0.1276$ by extra sum of squares, Ftest). $\mathbf{i}$ Relationship of the synaptic (slope $=0.8927, R^{2}=0.5828$ ) and non-synaptic (slope $=0.5755, R^{2}=0.3642$ ) GluA2 nanocluster numbers to the number of PSD-95 nanomodules per spine ( ${ }^{*} p=0.0143$, one-way ANCOVA, $n=167$ spines). Bars represent the mean \pm SEM.

spines containing synaptic AMPARs (>80\%) had non-synaptic nanoclusters of GluA2 subunit-containing AMPAR heterotetramers (Fig. 1f). Non-synaptic GluA2 nanoclusters were significantly smaller than synaptic GluA2 clusters (Fig. 1g, Avg. S-GluA2 size $=0.025 \mu \mathrm{m}^{2}$, Avg. NS-GluA2 size $=0.019 \mu \mathrm{m}^{2}$, $p<0.0001, \mathrm{~K}-\mathrm{S}$ test). Unlike synaptic GluA2 nanoclusters, the non-synaptic clusters were found in higher numbers and were less well related to PSD-95 nanomodule numbers (Fig. 1h, i). These data suggest that, unlike synaptic GluA2 heterotetramer nanoclusters, non-synaptic clusters are not modular.

Organization of AMPARs at spine synapses follows subunitspecific rules. GluA1 subunit-containing AMPAR heterotetramers can be incorporated into synapses with the expression of structural plasticity 5,30,31. The relationship between spine size, plasticity, and GluA1-containing AMPARs suggests that GluA1containing receptors represent a subset of AMPARs that do not follow a modular organization, allowing for more flexibility in the organization of spine synapses. The pattern of GluA1-containing AMPARs at spine synapses was visualized using antibodies against endogenous GluA1, and synaptic sites were determined by staining for endogenous PSD-95 and Bassoon (Fig. 3a and Supplementary Fig. 5). The majority of GluA1 subunits form heterotetramers with $\mathrm{GluA}_{2}^{2}$, therefore we expect that most GluA1 puncta would colocalize with GluA2. Consistent with this model, co-staining with antibodies against GluA1 and GluA2 subunits indicates that the majority of GluA1 nanoclusters colocalize with GluA2 clusters (90\%, Supplementary Fig. 4a-d). As expected, since GluA2 can tetramerize with both GluA1 and other AMPAR subunits (e.g., GluA3) ${ }^{27}$, only $\sim 50 \%$ of GluA2 subunit clusters at synapses colocalize with GluA1 (Supplementary Fig. 4d). These data suggest that AMPAR complexes containing GluA1 subunits and those lacking GluA1 subunits might not share the same nanoscale organization.

Consistent with the idea that AMPARs lacking GluA1 and those containing GluA1 behave differently at the nanoscale, only $53 \%$ of spines have GluA1-containing AMPAR puncta (Supplementary Fig. 4b). Within spines at sites marked by nanoclusters of PSD-95 and Bassoon, GluA2 colocalized with $81 \%$ of PSD-95 nanomodules (Supplementary Fig. 4a-c, GluA2 (A2) $=40 \%$, GluA1 and GluA2 $(\mathrm{A} 1+\mathrm{A} 2)=41 \%$, Total GluA2 $=81 \%$ of PSD-95 nanomodules). In contrast, GluA1 immunostaining was restricted to a subset of PSD-95 nanomodules (Supplementary Fig. $4 \mathrm{a}-\mathrm{c}$, GluA1 $(\mathrm{A} 1)=5 \%$, GluA1 and GluA2 $(\mathrm{A} 1+\mathrm{A} 2)=41 \%$, Total GluA1 $=46 \%$ of PSD-95 nanomodules). Of the $53 \%$ of spines that contained GluA1 immunostaining at synapses, $80 \%$ had only a single GluA1 nanocluster (Fig. 3a, b and Supplementary Fig. 5). In line with this skewed distribution, GluA1 cluster numbers did not scale as a function of PSD-95 nanomodule numbers. In spines with either one or two PSD-95 nanomodules, most spines contained only a single GluA1 nanocluster (Fig. 3c). Moreover, GluA1 cluster numbers scaled significantly less with spine size than PSD-95 and Bassoon, or GluA2 (S-GluA2 (Fig. 1e) vs. S-GluA1 (Fig. 3d), $p<0.0001$, one-way ANCOVA). Together these data suggest that GluA1-containing AMPARs might not respect the modular organization of adapter proteins.

GluA1 is linked to the expression of plasticity, suggesting that GluA1-containing AMPARs might be preferentially localized to larger spines while GluA2 heterotetramers might be more ubiquitously distributed ${ }^{5}$. To test whether GluA1 and GluA2 subunits may follow different organizational logic at spines, the distribution of spine sizes with single GluA2 or GluA1 clusters was compared to the distribution of spines with single PSD-95 clusters (Fig. 3e-h). Consistent with the model that GluA2 subunits form nanomodules that scale with spine size as a function of PSD-95 nanomodule numbers (Fig. 1d), neither the distribution of sizes nor the average size of GluA2-containing spines was different from spines containing the same number of PSD-95 nanomodules (Fig. 3e, f). In contrast, both the cumulative distribution of spine sizes and the average size of spines containing one GluA1 cluster were significantly larger than spines containing a single PSD-95 nanomodule (Fig. 3g, h). Indeed, the mean size of spines containing a single GluA1 nanocluster was similar to the size of spines with two PSD-95 nanomodules. These data are consistent with a model where GluA1-containing AMPARs are found preferentially in larger spines, while GluA2 has a distribution similar to PSD-95. Thus, overall GluA2-containing AMPARs respect the modular organization of PSD-95, while AMPARs with GluA1-subunits are preferentially localized to larger spines (Fig. 3i, j), consistent with the link between GluA1-containing AMPARs and synaptic plasticity $2,6,17$.

Non-synaptic GluA1 nanoclusters (Fig. 4a, b; open arrowheads in a) were similar to non-synaptic GluA2 clusters (Fig. 1g) as the average size of non-synaptic GluA1 nanoclusters was smaller than synaptic GluA1 clusters (Fig. 4c, Avg. S-GluA1 size $=0.019 \mu \mathrm{m}^{2}$, Avg. NS-GluA1 size $=0.016 \mu \mathrm{m}^{2}, p=0.0002$, two-tailed $\mathrm{K}-\mathrm{S}$ test). However, the proportion of all spines containing nonsynaptic GluA1 clusters was significantly lower than that observed for non-synaptic GluA2 clusters (Supplementary Fig. 6a, GluA1 $=54 \%$, GluA2 $=82 \%, p=0.0019$, one-way ANOVA). Of spines with non-synaptic GluA1, spines that contained both non- 


\section{a}
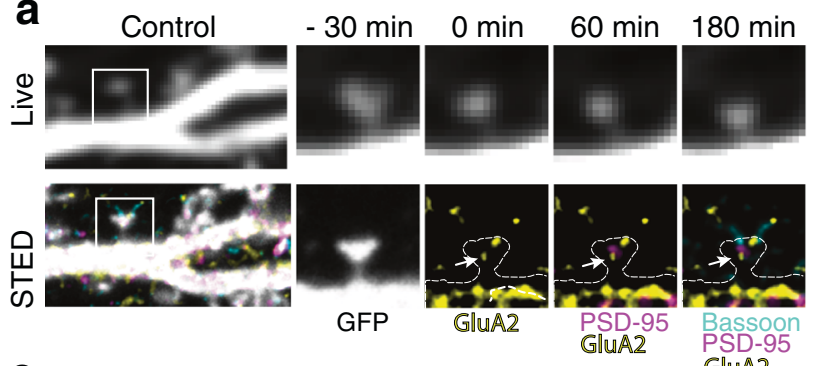

c
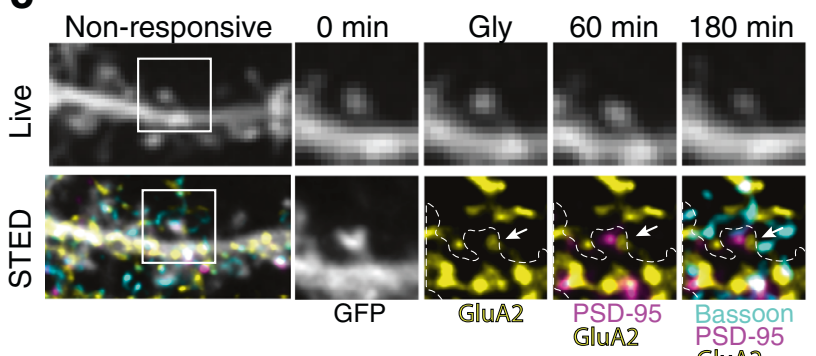

GluA2

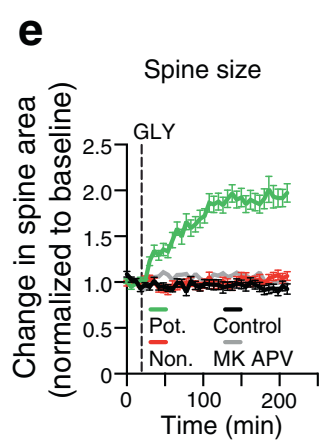

f

PSD-95 nanomodules (averages)

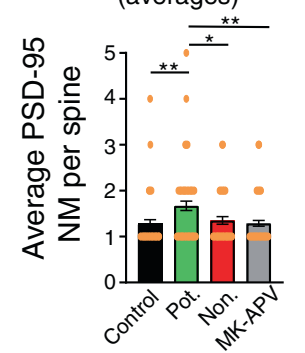

\begin{abstract}
g
\end{abstract}

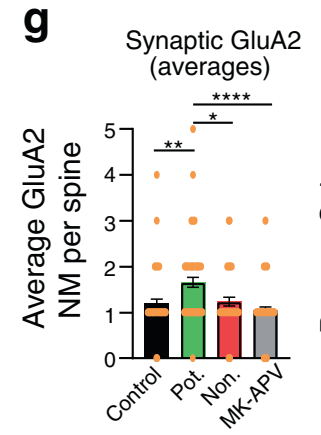

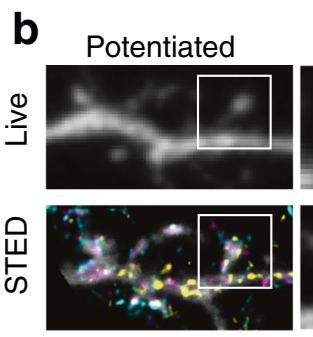

d

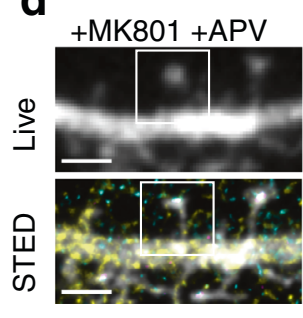

h
Synaptic GluA2
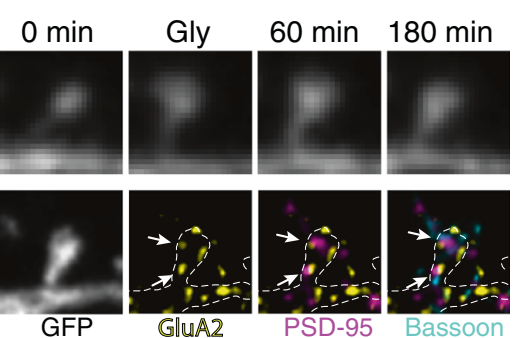

(distribution)
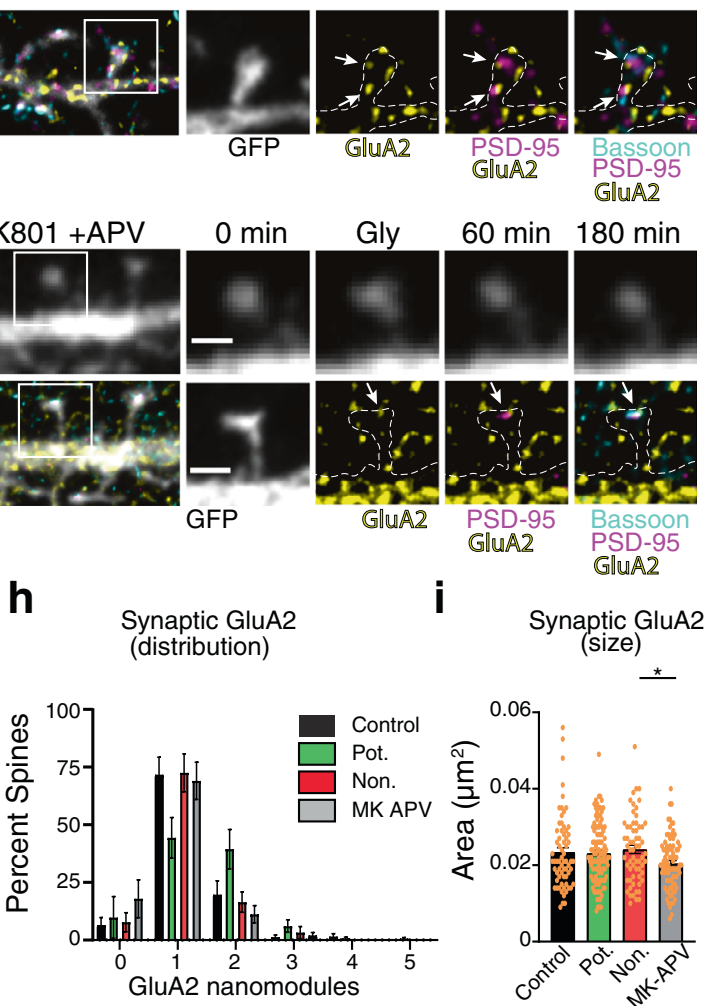

$60 \min 180 \mathrm{~min}$

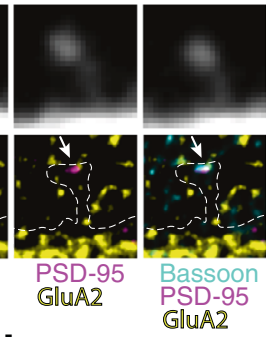

i

Synaptic GluA2 (size)

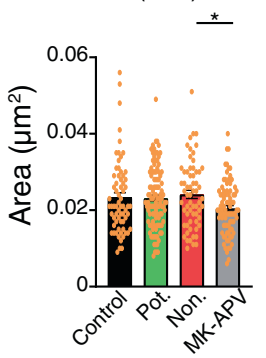

Fig. 2 NMDAR dependent structural plasticity is linked to increased number of synaptic GluA2 nanomodules. a-d Representative three-hour time-lapse images (top panels, confocal resolution) and post hoc STED images (bottom panels) of the same dendritic spines (white squares) of GFP-transfected DIV21-25 cortical neurons. Cells were retrospectively stained and imaged for cell morphology in confocal (gray, outline) and three-color STED of endogenous GluA2 (yellow, Atto-647N), PSD95 (magenta, Atto-425), and Bassoon (cyan, AlexaFluor-594). Arrows represent triple-colocalized GluA2/ PSD95/Bassoon clusters. e Quantification of change in spine area, normalized to baseline area, over three hours following treatment with glycine (3 min, $200 \mu \mathrm{M}$ ). Potentiated spines were defined by a sustained increase in spine area of $>10 \%$ over baseline (Pot., green traces, $n=64$ spines), non-responsive spines were defined as those that did not increase in spine area (Non., red traces, $n=51$ spines). Spine enlargement was blocked by treatment with $50 u M$ APV and $10 \mu \mathrm{M}$ MK-801 (gray traces, $n=79$ spines). Control spines were not subjected to glycine treatment (black traces, $n=56$ spines). Graph represents mean \pm SEM. $\mathbf{f}, \mathbf{g}$ Quantification of average number of PSD-95 (f) $\left(^{\star \star} p=0.002\right.$, one-way ANOVA with Tukey's post hoc) and synaptic GluA2 (g) ${ }^{\star \star \star \star} p<0.0001$, one-way ANOVA with Tukey's post hoc) modules per spine in the indicated conditions following retrospective STED imaging.

h Distributions of spines with single and multiple synaptic GluA2 modules in the indicated conditions, binned on the basis of the number of synaptic GluA2 clusters they contained. Conditions and spine numbers in (f-h) as in (e). i Quantification of the average area of individual synaptic GluA2 clusters in the indicated conditions (control: $n=67$ clusters; Pot: $n=106$ clusters; Non.: $n=63$ clusters; MK-APV: $n=83$ clusters; ${ }^{*} p=0.041$, one-way ANOVA, Tukey's posthoc). Bar graphs represent mean \pm SEM, with the number of individual spines or clusters represented by dots. ${ }^{\star} p<0.05,{ }^{\star \star} p<0.005,{ }^{\star \star \star} p<0.0001$. Scale bar for Live panel in d: $2 \mu \mathrm{m}$, applicable to Live panels in (a-d). Scale bar for STED panel in d: $2 \mu \mathrm{m}$, applicable to STED panels in (a-d). Scale bar for Live inset in $\mathbf{d}: 1 \mu \mathrm{m}$, applicable to Live insets in (a-d). Scale bar for STED inset in $\mathbf{d}: 1 \mu \mathrm{m}$, applicable to STED insets in (a-d).

synaptic and synaptic GluA1 nanoclusters were significantly larger than spines with only non-synaptic GluA1 nanoclusters (Fig. 4d). These data are consistent with a model where nonsynaptic GluA1 is positioned in smaller spines to be delivered to synaptic sites upon plasticity inducing stimuli $17,31,32$. Spines frequently contained either no non-synaptic GluA1 or different numbers of non-synaptic GluA1 puncta than would be expected from the number of PSD-95 puncta in the same spine. Thus, the number of non-synaptic GluA1-containing AMPAR nanoclusters found in spines was not well-related to PSD-95 nanomodule number or spine size (Fig. 4a-e, f; empty arrowheads in a). Indeed, of spines containing synaptic GluA1 nanoclusters (GluA1 puncta that colocalized with both PSD-95 and Bassoon) only $50 \%$ had non-synaptic GluA1 nanoclusters (Fig. 4b). These data indicate that non-synaptic GluA1 puncta are neither modular nor form structures that appear linked to the modular synaptic scaffold as defined by PSD-95.

Nanoscale organization of NMDARs in dendritic spine synapses. NMDARs are thought to be found in most spines, even in some spines that do not contain AMPARs ${ }^{3}$. At spines, NMDARs mediate the induction of synaptic plasticity and NMDAR activation induces increases in the number of pre- and postsynaptic adapter protein nanomodules that underlie structural plasticity ${ }^{25}$. Therefore, we examined whether NMDAR organization might correspond to the modular organization of PSD-95 in spines of different sizes. The nanoscale organization of the NMDAR in dendritic spines was visualized in EGFP-filled DIV2125 neurons by immunostaining with antibodies against the 
a

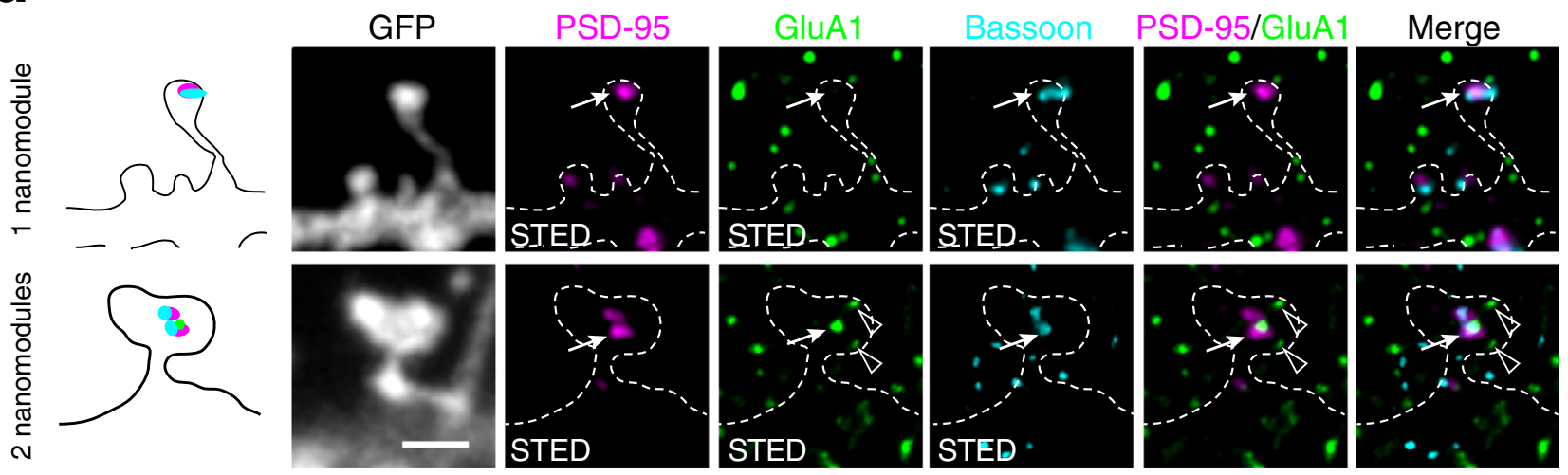

Synaptic GluA1 nanoclusters

b

PSD-95
GluA1

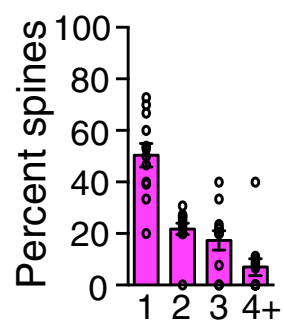

C

Bassoon
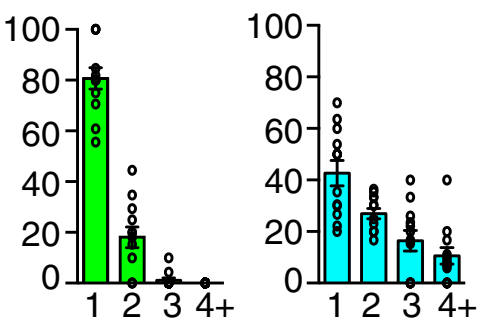

Number of nanomodules (only spines with GluA1)

e

Spine size

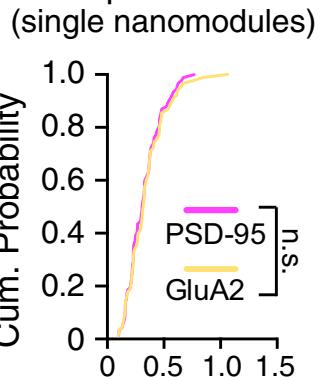

Spine size $\left(\mu \mathrm{m}^{2}\right)$
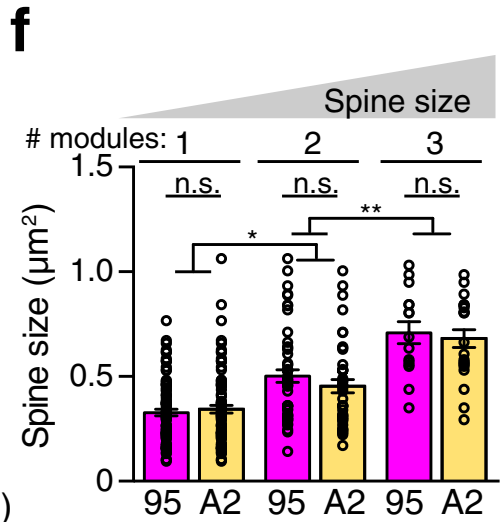

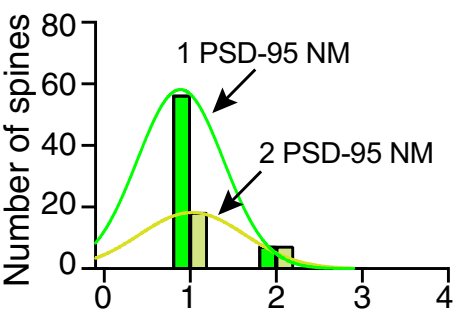

h d

Syn-GluA1 relationship to PSD-95 nanomodule number
Relationship to spine $\underset{\text { PSD-95 Bassoon S-GluA1 }}{\longrightarrow}$

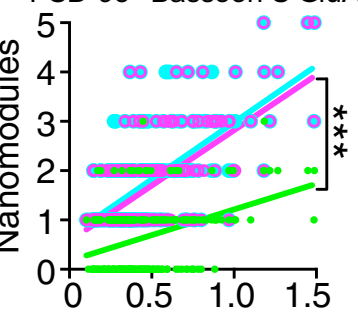

S-GluA1 nanoclusters/spine Spine size $\left(\mu \mathrm{m}^{2}\right)$

g

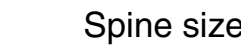

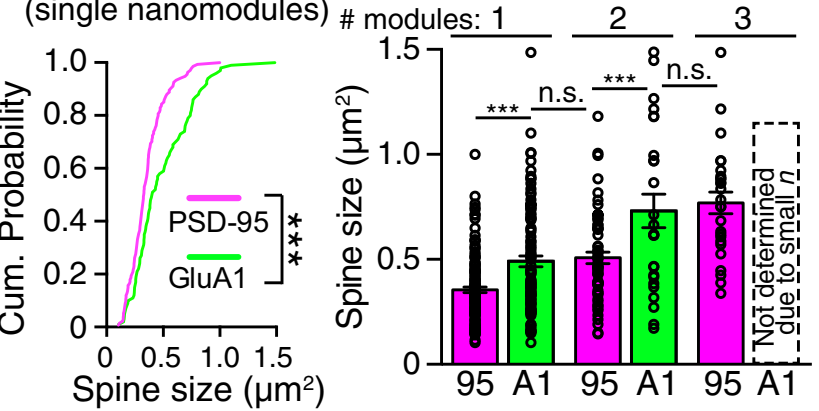

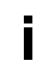

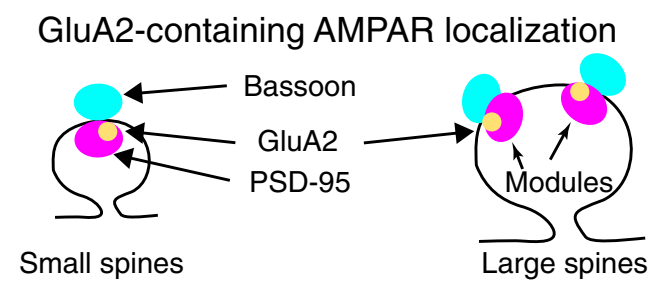

Small spines

endogenous GluN1 subunit of the NMDAR along with antibodies against endogenous PSD-95 and Bassoon (Fig. 5a and Supplementary Fig. 7). This approach enabled us to identify synaptic NMDARs which colocalized with both PSD-95 and Bassoon.

In cortical neurons, nearly all spines (96\%) contained synaptically-localized GluN1 subunits, indicating the presence of NMDAR heterotetramers at most synapses (Fig. 1b and j

GluA1-containing AMPAR localization

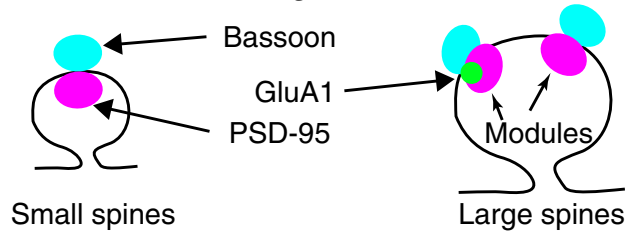

Supplementary Fig. $4 \mathrm{~b})$. Taken together with the GluA2 subunit distribution in spines (Fig. $1 \mathrm{~b}$ and Supplementary Fig. $4 \mathrm{~b}$ ) and PSD-95 nanomodules (Supplementary Fig. 4c), approximately 7\% of spines and $14 \%$ of PSD-95 nanomodules contain only NMDARs. These data are consistent with the observation that many spines lack AMPARs and might be 'silent' at resting membrane potentials ${ }^{7,8,27}$. 
Fig. 3 GluA1 containing AMPARs are found in large spines and are organized in a non-modular manner. a Images of single and multi-nanomodule spines from DIV21 GFP transfected cortical neurons stained for PSD-95 (magenta, Atto-425), GluA1 (green, Atto-647N), and Bassoon (cyan, AlexaFluor-594). Colocalization indicated by white arrows; arrowheads indicate GluA1 clusters alone. Scale bar: $1 \mu \mathrm{m}$. b Percentage of spines containing single and multiple PSD-95, GluA1, and Bassoon clusters ( $n=128$ spines). c Distribution of the number of synaptic GluA1 nanoclusters as a function of the number of PSD-95 nanomodules. Gaussian fits model two overlapping distributions of S-GluA1 puncta in one (green) and two (yellow) PSD-95 NM spines ( $p<0.0001$ by extra sum of squares, $F$ test), indicating that most spines contain only one synaptic GluA1 puncta, regardless of the number of PSD-95 puncta. d Quantification of the relationship between spine size $\left(n=240\right.$ spines) and the number of Bassoon (cyan line, $R^{2}=0.4076$, slope $=2.306 \pm 0.1802$ ), PSD-95 (magenta line, $R^{2}=0.4330$, slope $=2.246 \pm 0.1666$ ) and GluA1 (yellow line, $R^{2}=0.1515$, slope $=1.040 \pm 0.1596$, ${ }^{\star \star \star} p<0.0001$, one-way ANCOVA) nanopuncta. Comparison of PSD-95 and GluA2's relationship to spine size in spines with (e) single (PSD-95, $n=92$ spines, GluA2, $n=90$ spines, $p>0.9999$, two-tailed K-S test) and $\mathbf{f}$ multiple PSD-95 ( $n=52$ two-clustered spines, $n=15$ three-clustered spines) and GluA2 ( $n=42$ twoclustered spines, $n=21$ three-clustered spines, ${ }^{\star} p<0.018,{ }^{\star \star} p<0.034$, one-way ANOVA, Tukey's post hoc) clusters. Comparison of PSD-95 and GluA1's relationship to spine size in spines with (g) single (PSD-95, $n=134$ spines, GluA1, $n=100$ spines, ${ }^{\star \star \star} p=0.007$, two-tailed K-S test, ) and $\mathbf{h}$ multiple PSD95 ( $n=61$ two-clustered spines, $n=26$ three-clustered spines) and GluA1 ( $n=25$ two-clustered spines, ${ }^{\star \star \star} p<0.006$, one-way ANOVA, Tukey's post hoc) nanomodules. Model of GluA2 (i) and GluA1 (j) localization to small and large spines. Bars represent the mean \pm SEM. $P$ values for comparisons in $\mathbf{f}, \mathbf{h}$ are provided in the source data.

a

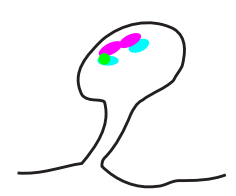

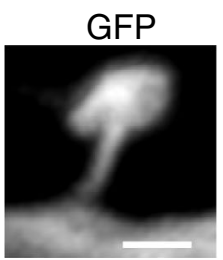
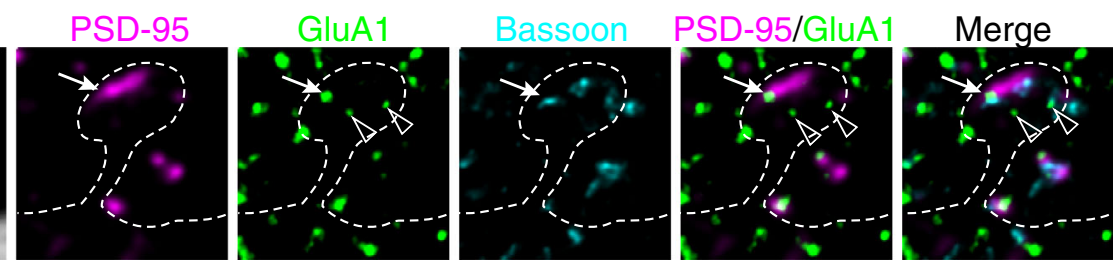

Non-synaptic GluA1 nanoclusters

b GluA1-containing

\section{C} spines
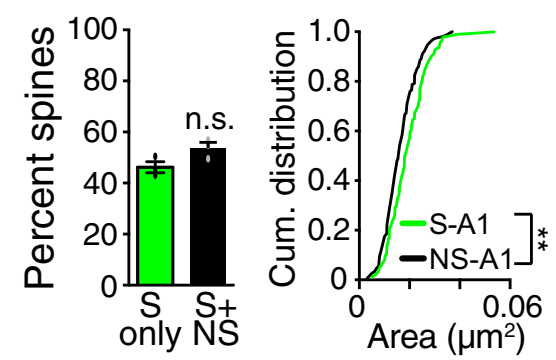

Nanocluster size

d

Spine size

e

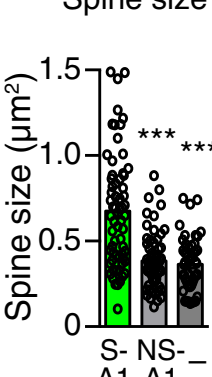

Non-Syn GluA1 relationship to $\mathrm{PSD}-95$ nanomodule number

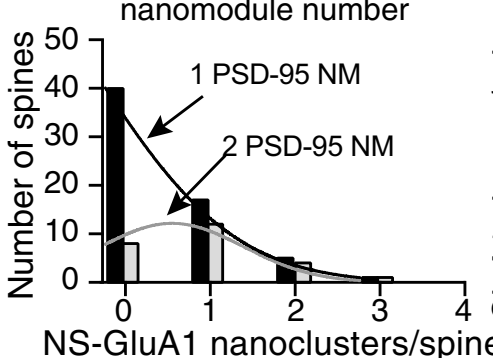

f

Syn. and Non-syn GluA1 relationship to PSD-95
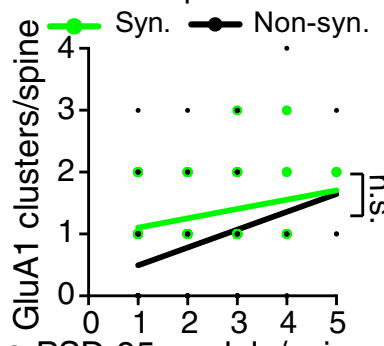

Fig. 4 Non-synaptic GluA1 clusters localize to small and large spines in a non-modular manner. a Image of a two-nanomodule spine from DIV21 GFP transfected cortical neuron stained for PSD-95 (magenta, Atto-425), GluA1 (green, Atto-647N), and Bassoon (cyan, AlexaFluor-594). White arrows indicate synaptic GluA1 nanoclusters; arrowheads indicate non-synaptic GluA1. Scale bar: $1 \mu \mathrm{m}$. b Percent of spines containing only synaptic (green bar, $n=112$ spines) or both, synaptic and non-synaptic (black bar, $n=125$ spines) GluA1 nanoclusters ( $p=0.07$, two-tailed Student's t-test, dots represent averages of data from three individual experiments). c Cumulative probability distributions of synaptic and non-synaptic GluA2 nanocluster sizes ( ${ }^{\star \star} p=0.004$, two-tailed K-S test). d Average spine size of spines containing both non-synaptic and synaptic GluA1 nanoclusters (S-A1, $n=65$ spines), only non-synaptic GluA1 (NS-A1, $n=60$ spines), or lacking GluA1 containing AMPARs $\left(-, n=50\right.$ spines, ${ }^{\star \star \star} p<0.0001$, one-way ANOVA with Tukey's post hoc). e Distribution of the number of non-synaptic GluA1 nanoclusters relative to the number of PSD-95 nanomodules per spine (One PSD-95 NM spines black, Two PSD-95 NM gray). Gaussian fits were used to model non-synaptic GluA1 nanocluster distribution ( $p<0.0001$ by extra sum of squares, F test). The number of NS-GluA1 puncta was not related to PSD-95 puncta number. $\mathbf{f}$ Relationship of the synaptic $\left(\right.$ slope $\left.=0.1524, R^{2}=0.1204\right)$ and nonsynaptic (slope $=0.2878, R^{2}=0.1164$ ) GluA1 nanocluster numbers to the number of PSD-95 nanomodules per spine ( $p=0.086$, one-way ANCOVA, $n=127$ spines, only spines containing synaptic GluA1 were included). Bars represent the mean \pm SEM.

The NMDAR receptor complex interacts with PSD- $95^{33}$. Therefore, we asked if the distribution of GluN1 puncta in spines resembled that of PSD-95 and Bassoon. The fraction of spines with one, two, three, or four synaptic GluN1 puncta was similar to the number of PSD-95 and Bassoon nanomodules, suggesting that GluN1 might also form modules (Fig. 5a, b and Supplementary Fig. 7). Consistent with this model, the number of synaptic GluN1 nanoclusters varied with the number of PSD-95 nanomodules (Fig. 5c). Moreover, the number of synaptic GluN1 clusters scaled linearly with spine size (Fig. 5d). These data suggest that GluN1 forms synaptic nanomodules that scale in number as spine size increases.

Similar to GluA1, approximately $40 \%$ of spines contain GluN1 clusters that do not colocalize with synaptic markers (Fig. 5a-e; open arrowheads in 3a, 4a, and Supplementary Fig. 6a). These non-synaptic GluN1 nanoclusters were significantly smaller in size than synaptic GluN1 nanoclusters (Fig. 5f, Avg. S-GluN1 size $=0.036 \mu \mathrm{m}^{2}$, Avg. NS-GluN1 size $=0.026 \mu \mathrm{m}^{2}, p<0.0001$, 
a

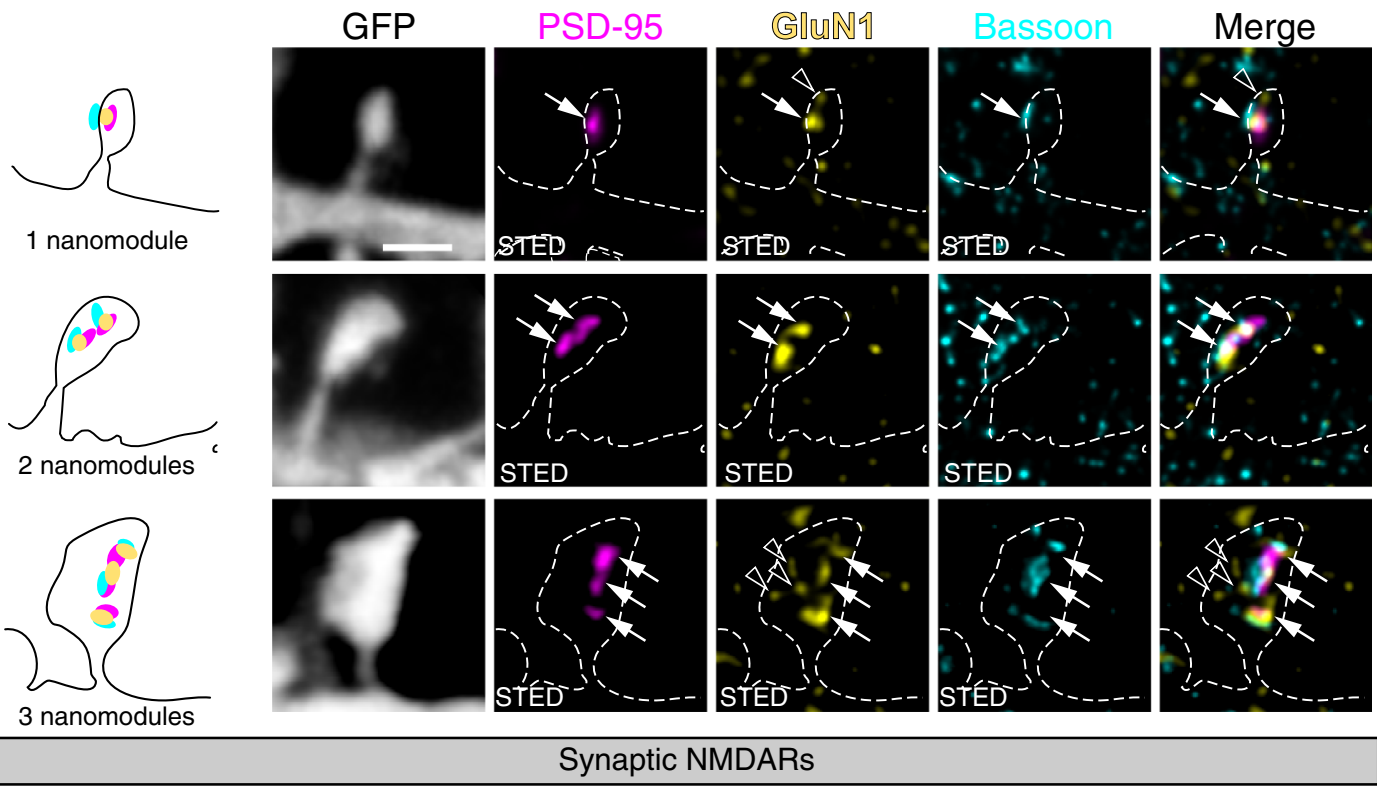

b
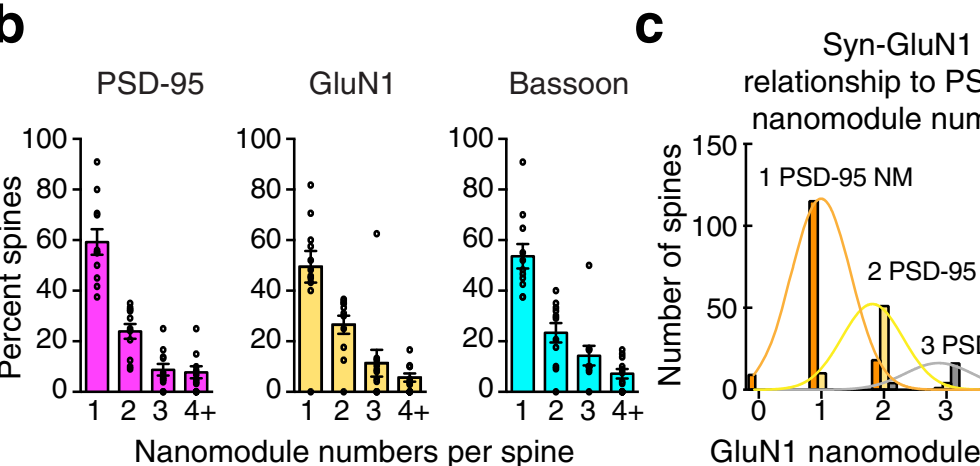

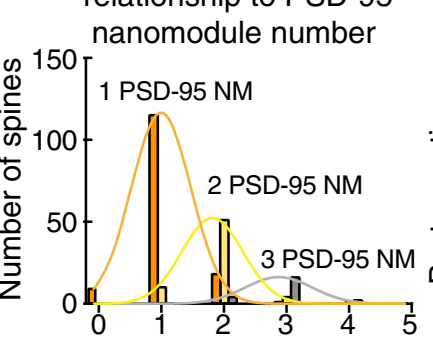

GluN1 nanomodule per spine

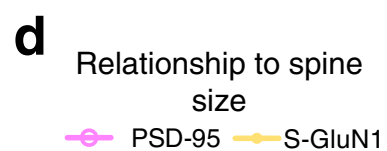

PSD-95 $=$ S-GluN1

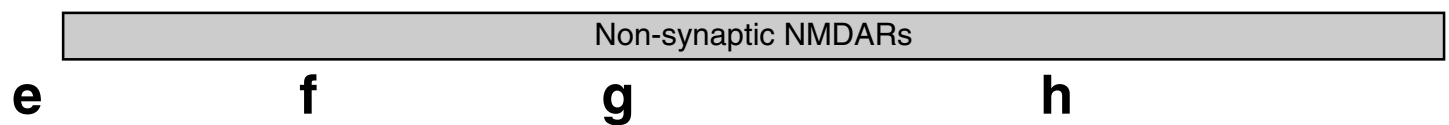

GluN1-containing Nanocluster size
spines
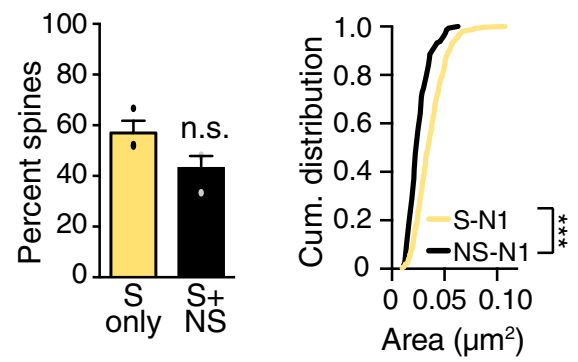

Non-syn GluN1

Relationship to PSD-95

nanomodule number

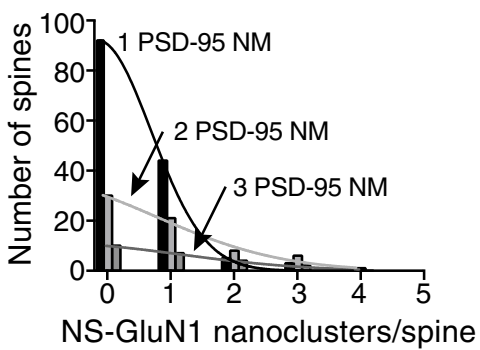

Syn. and Non-syn GluN1

relationship to PSD-95

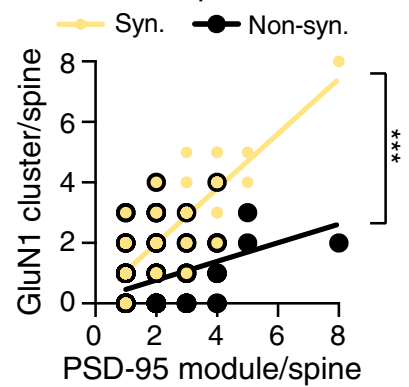

two-tailed $\mathrm{K}-\mathrm{S}$ ). The number of non-synaptic nanoclusters was poorly linked to PSD-95 nanomodule numbers (Fig. 5g, h), with no clear relationship to spine size (Supplementary Fig. 6b). Together these data indicate that in contrast to non-synaptic GluN1 nanoclusters, synaptic GluN1 subunits scale in number as spine size increases, suggesting that they are organized in a modular fashion.

Subunit-specific organization of NMDARs within spine synapses. The synaptic content of GluN2A and GluN2B NMDAR subunits changes the ability of a synapse to undergo structural plasticity, suggesting that the specific ratio of GluN2A to GluN2B

in NMDAR heterotetramers may tune synaptic function ${ }^{1,9,10}$. Therefore, we examined the distribution of endogenous GluN2A and GluN2B in spines relative to PSD-95 nanomodules (Figs. 6a, 7a and Supplementary Fig. 8). Analysis of the number of GluN2 clusters in spines suggests a more flexible relationship to spine size than seen for the obligatory GluN1 subunit, likely reflecting distinct functional roles of GluN2 subunits in developing (GluN2B) and mature (GluN2A) spines $9,34,35$. Consistent with this more flexible organization, the pattern of GluN2 subunits in spines varied. An array of different combinations of GluN2 subunit distributions were found: from spines with only GluN2A or only GluN2B subunits (Fig. 6a and Supplementary 
Fig. 5 Nanoscale organization of NMDARs in dendritic spine synapses reflects the modular organization of pre- and postsynaptic adaptor molecules. a Representative images of single, two, and three nanomodule spines from DIV21 cortical neurons. GFP (gray, dotted lines) was used to visualize spine morphology in confocal mode. Antibodies to PSD-95 (magenta, Atto-425), GluN1 (yellow, Atto-647N), and Bassoon (cyan, AlexaFluor-594) were used to identify adapter protein nanomodules and NMDARs in STED mode (arrows). Open arrowheads indicate non-synaptic GluN1 nanoclusters. Scale bar: $1 \mu$ m. b Percentage of spines containing single and multiple PSD-95, GluN1, and Bassoon clusters ( $n=251$ spines). c Distribution of the number of synaptic GluN1 nanoclusters as a function of PSD-95 nanomodule numbers per spine. Gaussian fits model distinct distributions of S-GluN1 puncta in one (orange), two (yellow), and three (gray) PSD-95 NM spines. The three distributions were non-overlapping and could not be fit with a single distribution ( $p<0.0001$ by extra sum of squares, $\mathrm{F}$ test). $\mathbf{d}$ The number of PSD-95 and synaptic GluN1 nanomodules scales linearly with spine size indistinguishable from each other (PSD-95-magenta line, $R^{2}=0.4138$, slope $=2.015 \pm 0.1523$; GluN1-yellow line, $R^{2}=0.3326$, slope $=1.902 \pm 0.1711, p=0.8364$, one-way ANCOVA,

$n=250$ spines). e Percent of spines containing only synaptic (yellow bar, $n=137$ spines) or both, synaptic and non-synaptic (black bar, $n=114$ spines) GluN1 nanoclusters ( $p=0.6806$, two-tailed Student's t-test, dots represent averages of data from three individual experiments). $\mathbf{f}$ Cumulative probability distributions of synaptic and non-synaptic GluN1 nanocluster sizes ( ${ }^{\star \star *} p<0.0001$, two-tailed K-S test). $\mathbf{g}$ Distribution of the number of non-synaptic GluN1 nanoclusters relative to the number of PSD-95 nanomodules per spine as in (c). Distributions were overlapping with the same means for both data sets ( $p=0.3990$ by extra sum of squares F-test). Gaussian fits model distinct distributions of NS-GluN1 puncta in one (black), two (light-gray), and three (darkgray) PSD-95 NM spines. The number of NS-GluN1 puncta was not related to PSD-95 puncta number. h Relationship of synaptic (slope $=0.9076$, $\left.R^{2}=0.7346\right)$ and non-synaptic (slope $\left.=0.3076, R^{2}=0.3076\right)$ GluN1 nanocluster numbers to the number of PSD-95 nanomodules per spine $(p<0.0001$, one-way ANCOVA, $n=249$ spines). Bars represent the mean \pm SEM.

Fig. 8a-d), to spines with both GluN2A and GluN2B subunits either in separate nanoclusters or in the same nanocluster (Fig. 7a, cyan, and yellow arrows, and Supplementary Fig. 8e, f, also see Fig. $7 \mathrm{~b}-\mathrm{d}$ binary image insets). These observations suggest that GluN2 nanoclusters might not follow a strict modular organization with spine size increases.

Nearly all spines (96\%) contained the obligatory GluN1 subunit at synapses (Fig. $1 \mathrm{~b}$ and Supplementary Fig. 4b), but the distribution of endogenous GluN2 subunits was heterogeneous. Approximately $25 \%$ of spines contained only GluN2A, 20\% contained only GluN2B, while $45 \%$ contained both GluN2A and GluN2B (Fig. 6b). To define the relationship between NMDAR subtypes and spine size, we determined the distribution of synaptic GluN2 puncta numbers in individual spine synapses. Similar to GluN1 and PSD-95, we found that approximately $65 \%$ of spines that contained GluN2A puncta had a single GluN2A cluster, 25\% had two, and less than 5\% had three or more GluN2A clusters (Fig. 6c). In contrast, $83 \%$ of spines with GluN2B puncta contained a single GluN2B cluster (Fig. 6d). These data suggest that nanoscale clusters of NMDARs that contain GluN2B subunits might not scale with spine size.

Analysis of spines with only GluN2A nanoclusters revealed that the number of GluN2A clusters increased with spine size (Fig. 6e, $p<0.0001$, Pearson's correlation). However, consistent with a more flexible relationship to spine size for the GluN2 subunits, GluN2A-containing NMDARs scaled significantly less well with spine size than PSD-95 ( $p=0.0138$, one-way ANCOVA), likely due to many larger spines that contained both GluN2A and GluN2B subunits (Fig. 7d). In contrast, analysis of the relationship between spine size and GluN2B nanocluster numbers in spines containing only GluN2B-containing NMDARs revealed a negative correlation with spine size (Fig. 6 e, $p=0.01$, Pearson's correlation). Moreover, the distribution of spine sizes indicates that spines containing single puncta of GluN2B are significantly smaller than those that contain single puncta of PSD-95 or GluN2A (Fig. 6f, g, $p=0.04$, two-tailed K-S test). These data suggest that as spines become larger, they are less likely to contain only GluN2B-containing NMDAR heterotetramers.

In spines that contained both GluN2A and GluN2B nanoclusters, the total number of clusters was positively correlated and increased with spine size (Fig. 7b). Consistent with the model that GluN2B cluster numbers do not increase with spine size, most spines contained only a single GluN2B nanocluster (Fig. 7b inset), skewing the relationship toward GluN2A (GluN2A: $p<0.0001, \mathrm{~F}-$ statistics, GluN2B: $p=0.28$, F-statistics). These data suggest that when present in larger spines, GluN2B-containing NMDARs tend to be localized to a single nanocluster, while the number of GluN2A-containing NMDAR nanoclusters increases with spine size.

We next examined whether GluN2 subunits formed separate clusters or were found together at synapses. In smaller spines with single nanomodules of PSD-95, approximately equal proportions of spines (25-35\%) had clusters of GluN2A alone, GluN2B alone, or both GluN2A and GluN2B (Fig. 7c and Supplementary Fig. $9 \mathrm{a}-\mathrm{c}$ ). These data suggest that at single PSD-95 nanomodules, GluN2A and GluN2B clusters can co-localize. Consistent with these data, $60 \%$ of the larger spines with two PSD-95 nanomodules contained both GluN2A and GluN2B while a minority $(8.7 \%)$ of the two nanomodule spines contain GluN2B only (Fig. 7d). Analysis of individual PSD-95 nanomodules in these larger spines indicates that a higher proportion of nanomodules colocalize with both GluN2A and GluN2B than in smaller single nanomodule spines (37\% vs. $25 \%$, Supplementary Fig. 9c, d). To determine how GluN2 subunits localize within the context of the modular organization of the synapse, we examined PSD-95 nanomodules that co-localize with both GluN2 subunits in spines. At these spine-localized PSD-95 nanomodules, the majority of GluN2A and GluN2B puncta overlap (86\%). Interestingly, the overlap was not complete, with an average area of colocalization of only $29.7 \%$ (Supplementary Fig. 9e, f). Together with data that GluN2A and GluN2B subunits segregate within the synapse ${ }^{36}$, our data suggest that GluN2 subunits follow subunit-specific rules that govern their organization in spines. GluN2B subunits are found in the smallest spines, while GluN2A-containing NMDARs form nanomodules that scale with spine size. These data are consistent with the distinct functions of GluN2B containing NMDARs during development and the induction of plasticity, and GluN2A NMDAR heterotetramers in the mature brain and after plasticity $3,9,10,37,38$. It is important to note that the more flexible pattern of GluN2 subunit organization is built on the precise modular organization of the GluN1 subunit, which forms nanomodules that scale in a fashion indistinguishable from PSD-95. Thus, NMDAR organization reflects a well-organized and robust synaptic nanostructure while providing a flexible architecture (Fig. 7e)

NMDARs and AMPARs exhibit distinct nanoscale relationships to PSD-95 nanomodules. The relationship between glutamate receptors and PSD-95 at spine synapses has been the 
a

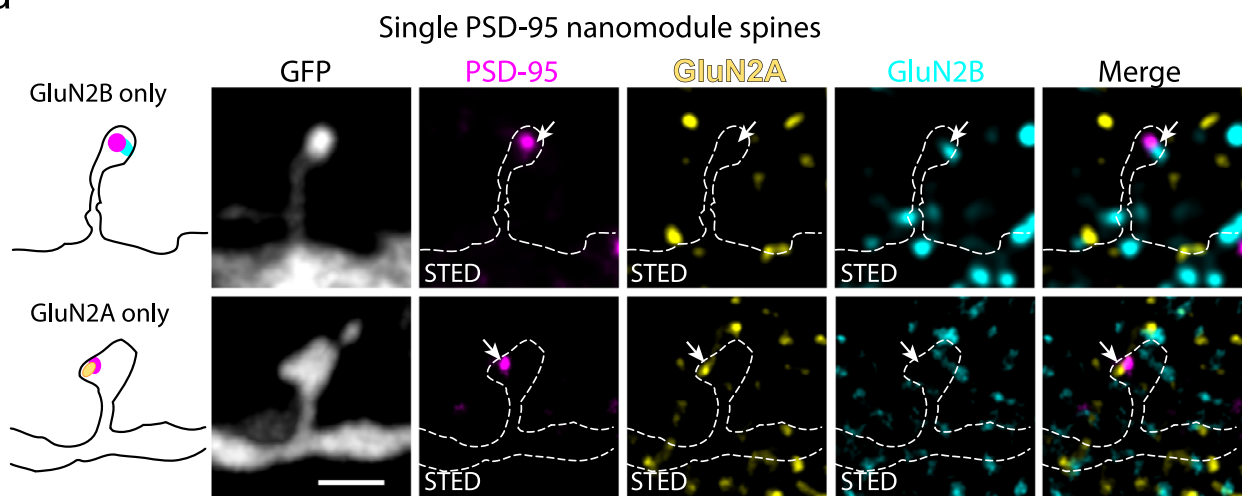

b

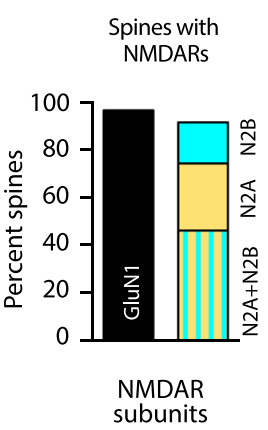

e

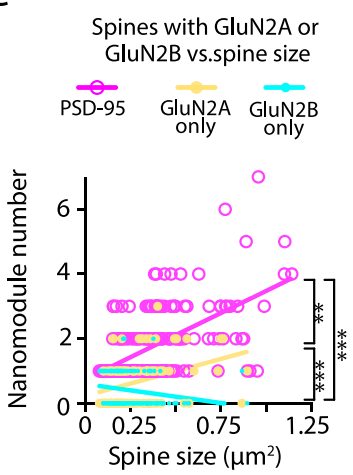

C
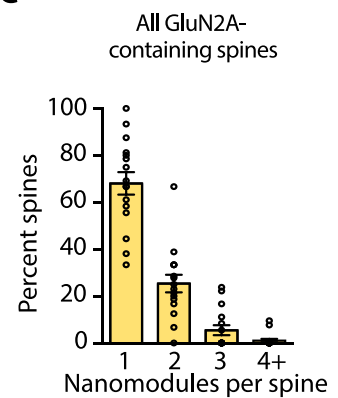

f
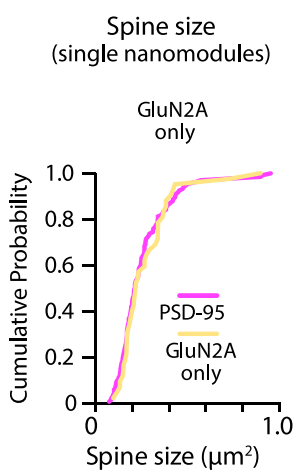

d

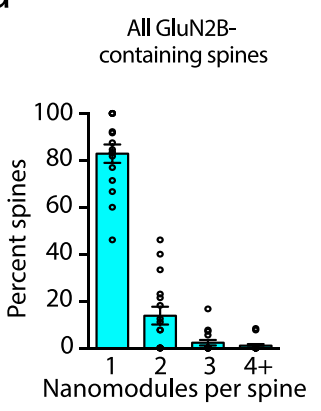

9

Spine size

(single nanomodules)

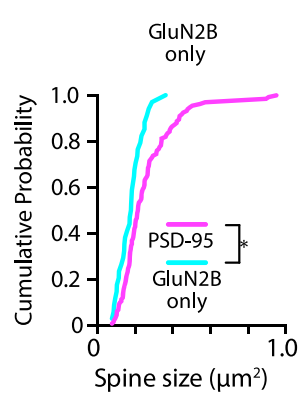

Fig. 6 The nanoscale organization of GluN2A and GluN2B NMDAR subtypes in dendritic spine synapses with single PSD-95 nanomodules. a Threecolor STED images of a single PSD-95 nanomodule (magenta, Atto-425) spine with colocalized GluN2A (yellow, AlexaFluor-594) and GluN2B (cyan, Atto$647 \mathrm{~N}$ ) clusters. Arrows indicate PSD-95 nanomodules containing only GluN2A or only GluN2B. Scale bars: $1 \mu$ m. b Percent of spines containing NMDAR subunits ( $G$ luN1 $=242 / 251$ spines, GluN2A + GluN2B $=112 / 239$ spines, GluN2A only $=67 / 239$ spines, GluN2B only $=40 / 239$ spines). , $\mathbf{d}$ Percent of spines with single and multiple GluN2A ( $n=175$ spines) and GluN2B ( $\mathbf{d} n=154$ spines) cluster numbers per spines. e Plots of the relationship between spine size and the number of PSD-95 (magenta, $R^{2}=0.3391$, slope $=2.728 \pm 0.2473$ ), GluN2A only (yellow, $n=132$ spines, $R^{2}=0.1179$, slope $=1.522 \pm 0.3652$ ) and GluN2B only nanomodules (cyan, $n=127$ spines, $R^{2}=0.0508$, slope $=-0.7803 \pm 0.3015 ;{ }^{\star \star \star} p<0.0001,{ }^{\star \star} p=0.0138$, one-way ANCOVA). f, $\mathbf{g}$ Cumulative probability distributions as a function of spine size in spines with a single PSD-95 nanomodule (magenta, $n=133$ spines) vs. spines with a single GluN2A cluster only (f yellow, $n=42$ spines, $p=0.9466$, two-tailed K-S test) or GluN2B cluster only ( $\mathbf{g}$ cyan, $n=34$ spines, ${ }^{\star} p=0.04$, two-tailed K-S test). Bars represent the mean \pm SEM.

subject of intense interest ${ }^{39-45}$. To begin to address this relationship at the nanoscale, we measured the distances between centers of STED-resolved clusters of endogenous synaptic PSD-95 (juxtaposed to Bassoon) and colocalized nanoclusters of either NMDARs (GluN1) or AMPARs (GluA2 or GluA1; Fig. 8a-c and Supplementary Fig. 4e, f). Segmentation and subsequent quantification were performed on XYZ super-resolved nanoclusters of synaptically localized proteins within the entire imaged field (See "Methods" and Fig. 8d) $)^{25,46}$. As expected, there were no significant differences between GluA1 and GluA2 subunitcontaining AMPAR localization relative to PSD-95 (Fig. 8e, f and Supplementary Fig. 4e, f). Similar results were obtained using two different combinations of secondary antibodies (PSD95Atto-425, Bassoon- AlexaFluor-594, and GluA1- Atto 647NFig. 8c, and GluA1- Atto-425, PSD95- AlexaFluor-594, and Basson- Atto-647N Supplementary Fig. 10) suggesting that these findings were not due to chromatic aberration or the mix of secondary antibodies used (Supplementary Figs. 1 and 10). In contrast, the centers of PSD-95 nanomodules were significantly closer to the centers of GluN1 subunit nanoclusters than to either GluA1 or GluA2 subunit clusters both on average and over the distribution (GluN1: $173.4 \pm 2.0 \mathrm{~nm}$; GluA1: $189.1 \pm 3.0 \mathrm{~nm}$; 
a

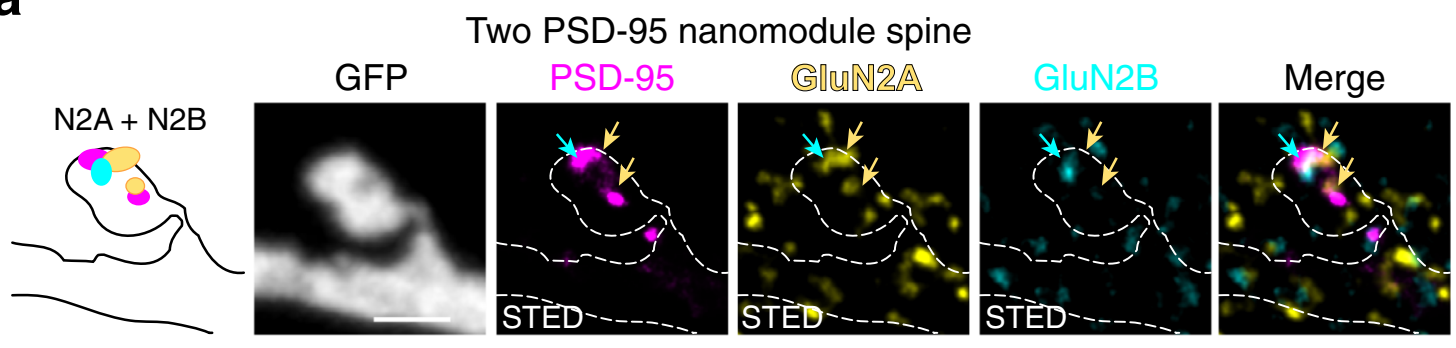

b

Spines with GluN2A and GluN2B vs. spine size

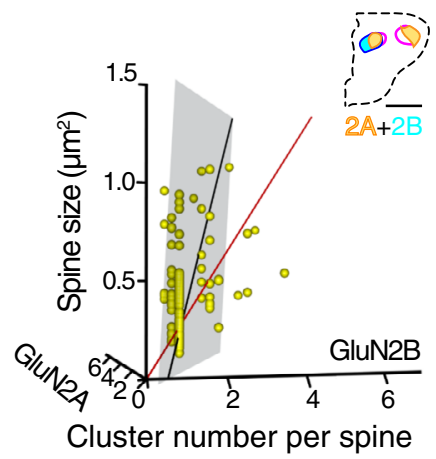

C

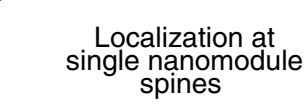

d

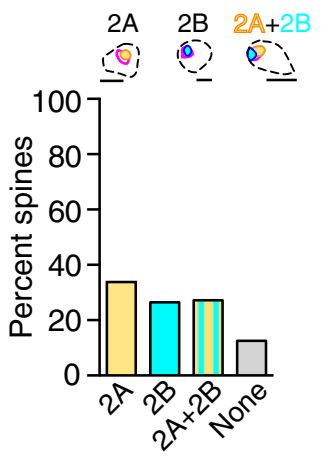

$$
\begin{gathered}
\text { Localization at } \\
\text { two nanomodule } \\
\text { spines }
\end{gathered}
$$

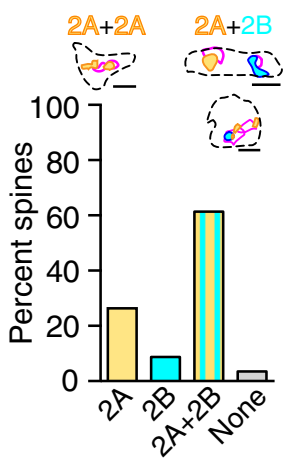

e

Fig. 7 The nanoscale organization of GluN2A and GluN2B NMDAR subtypes in dendritics spines with multiple PSD-95 nanomodules. a Three-color STED image of a two PSD-95 nanomodule (magenta, Atto-425) spine with colocalized GluN2A (yellow, AlexaFluor-594) and GluN2B (cyan, Atto-647N) clusters. Arrows indicate PSD-95 nanomodules containing GluN2B (cyan arrow) and/or GluN2A (yellow arrow). Scale bars: $1 \mu$ m. b A multivariable linear regression of spine size as a function of the number of GluN2A ( $p=6.34 \times 10^{-9}$, F-statistics) and GluN2B ( $p=0.28$, F-statistics) clusters in spines containing both GluN2A and GluN2B ( $n=110$ spines). Regression plane (gray square) and the best fit line (black) were determined by the coefficients for GluN2A (0.417 \pm 0.06$)$, GluN2B $(0.26 \pm 0.23)$, and the intercept $(0.53 \pm 0.11)$. The line of unit slope is shown in red. Inset: An example of the most commonly occurring localizations of GluN2A clusters (yellow) and GluN2B clusters (cyan) relative to PSD-95 nanomodules (magenta) in spines containing both types of GluN2 clusters. A binary image is shown. Spine head area is outlined by a dotted line. Scale bar: $500 \mathrm{~nm}$. Distribution of NMDAR subunits in spines with $\mathbf{c}$ single ( $n=136$ spines) and $\mathbf{d}$ two ( $n=57$ spines) PSD-95 nanomodules. Insets: Binary image examples of GluN2A (yellow) and GluN2B (cyan) nanocluster localizations in spines with $\mathbf{c}$ one and $\mathbf{d}$ two PSD-95 nanomodules (magenta). Spine head area is outline by a dotted line. Scale bar: $500 \mathrm{~nm}$. e Model of GluN2A and GluN2B cluster distribution in small and large spines. Bars represent the mean \pm SEM.

GluA2: $186.1 \pm 2.1 \mathrm{~nm}$; Fig. 8e, f). Similarly, both GluN2A and GluN2B subunit nanoclusters were about the same distance from PSD-95 as GluN1 and closer to PSD-95 than GluA1 (GluN2B: $179.7 \pm 2.0 \mathrm{~nm}$; GluN2A: $177.0 \pm 2.5 \mathrm{~nm}$; Supplementary Fig. 9h, i). GluN2A subunits were closer than GluA2 subunits to PSD-95 (Supplementary Fig. 9j). These data indicate that AMPARs and NMDARs are localized to different synaptic sub-diffraction domains at individual synapses, where NMDARs are preferentially localized closer to the centers of PSD-95 and AMPARs are at the periphery of PSD-95 nanomodules (Fig. 8g; Supplementary Fig. 9a, b and h, i, j). This link between glutamate receptor modularity and spatial organization suggests that glutamate receptors are organized to optimize synaptic function $13,20,39$.

NMDARs and AMPARs are distributed to distinct synaptic nano-domains. Among the molecules required for vesicle fusion, the presynaptic calcium sensors play a central role in regulating vesicle release kinetics ${ }^{47}$. The presence of the fast calcium sensor Synaptotagmin 1 (SYT1) on the membrane of synaptic vesicles is thought to underlie rapid vesicle fusion with the presynaptic membrane at the majority of central synapses ${ }^{47,48}$. Beyond rapid vesicle fusion, efficient synaptic transmission depends on the nanometer alignment between sites of vesicle fusion and glutamate receptors. These data suggest that the nanoscale positioning of synaptic glutamate receptors relative to glutamate release sites will impact synaptic function ${ }^{1,20,21,49}$. Yet, how SYT1 is organized in individual active zones and SYT1's position relative to NMDARs and AMPARs are not known.

To begin to resolve this issue, we tested whether SYT1 might respect the modular rules of the adapter proteins and glutamate receptors by defining the relationship between presynaptic SYT1 and spine size. We stained DIV21-25 neurons with antibodies for endogenous PSD-95, Bassoon, and SYT1 (Fig. 9a). This allowed us to identify SYT1 that colocalized with Bassoon juxtaposed to PSD-95 (Fig. 9b). Consistent with the importance of SYT1 for synaptic transmission, we found that SYT1 colocalized with Bassoon at $97 \%$ of spines, and SYT1 clusters co-distributed with Bassoon and PSD-95 nanomodules at synapses (Fig. 9c). Notably, the number of SYT1 puncta scaled with spine size indistinguishable from Bassoon nanomodules (Fig. 9d). These data indicate that SYT1 follows the rules of modularity at excitatory synapses ${ }^{25}$.

What is SYT1's nanoscale relationship to AMPARs and NMDARs? One possibility is that SYT1 could be localized at the active zone to match the positioning of the AMPAR in the PSDs. Such nanoscale arrangement might be necessary for efficient activation of AMPARs that have low affinity for glutamate ${ }^{1,21,49}$. The presence of such functionally specialized zones within a single synapse is suggested by both spatiotemporal modeling of glutamate in the cleft and the flexible nanoarchitecture of active zone and 

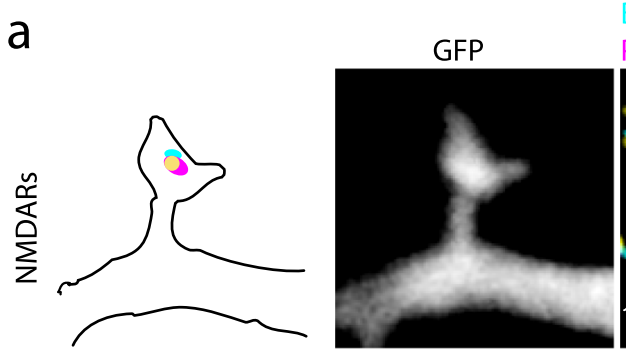

Bassoon

a

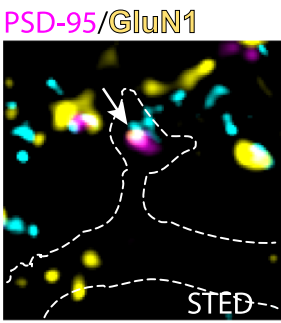

PSD-95/GR凹N1

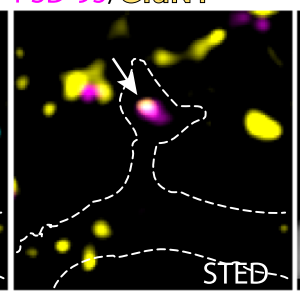

$3 \mathrm{D}$ rendering
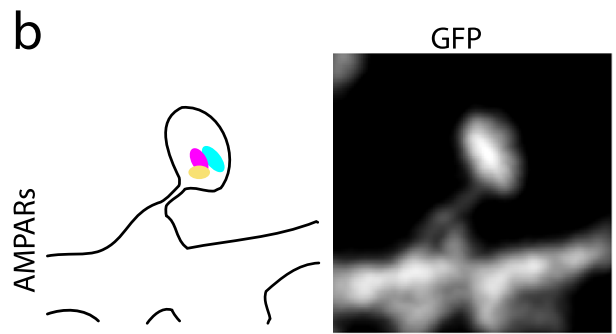

Bassoon

C
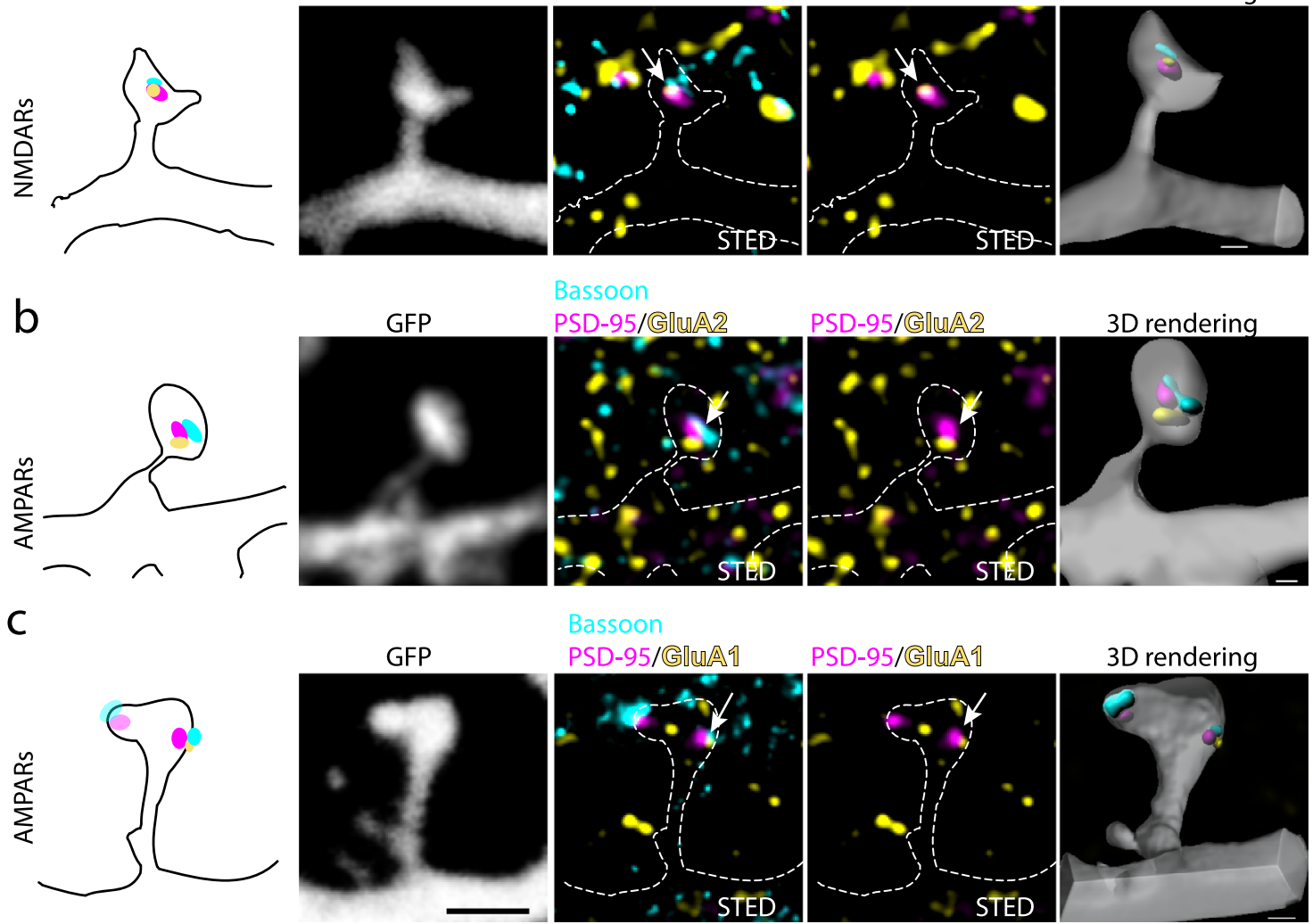

Bassoon
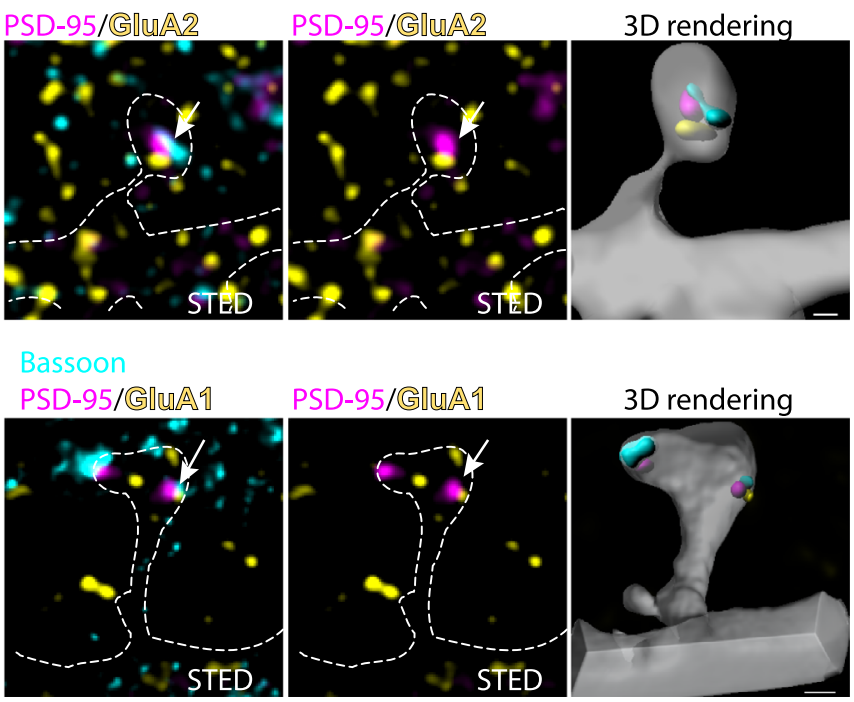<smiles>[AlH2]</smiles>

e

Distance from PSD-95
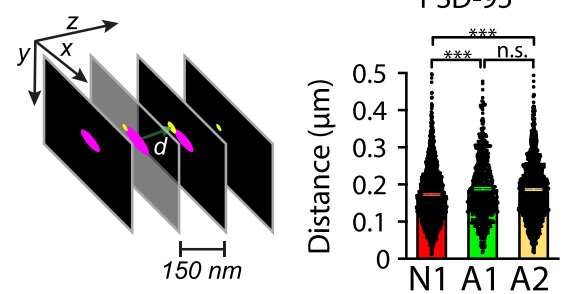

Center PSD-95 to center GluR distribution

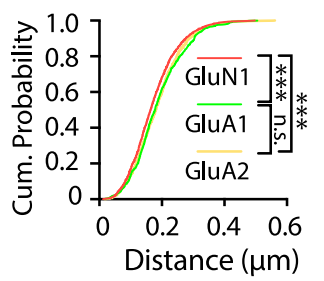

g

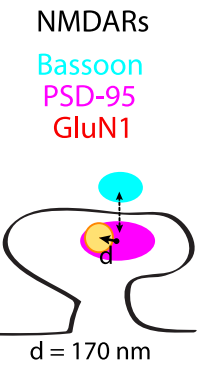

$3 \mathrm{D}$ rendering

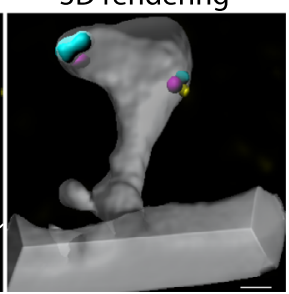

Model

AMPARs

Bassoon

PSD-95

GluA1 or GluA2

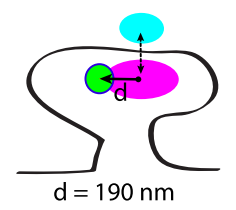

Fig. 8 NMDARs and AMPARs are localized to distinct parts of PSD-95 nanomodules. Representative images of one (a, b) and two (c) nanomodule spines from DIV21 cortical neurons. Antibodies to PSD-95 (magenta, Atto-425) and Bassoon (cyan, AlexaFluor-594) were used to identify synaptic nanomodules in STED mode in GFP-labeled spines (gray, dotted line, confocal mode). Arrows indicate synaptic a GluN1 nanoclusters (yellow, Atto-647N), b GluA2 nanoclusters (yellow, Atto-647N) and c GluA1 nanoclusters (yellow, Atto-647N). Scale bar for projections: $1 \mu \mathrm{m}$ (a-c). 3D rendering of indicated glutamate receptor subunit localization relative to PSD-95/Bassoon nanomodules is shown on the right. Scale bars for rendered images: $200 \mathrm{~nm}$ (a, b) and $300 \mathrm{~nm}(\mathbf{c})$. d Schematic of the segmentation and distance measurements ( $d$, green arrow) between the centers of pre- and postsynaptic clusters that were STED resolved in $X Y(\sim 50 \mathrm{~nm})$ and $Z(\sim 300 \mathrm{~nm})$. e Averages ( ${ }^{\star \star *} p<0.0001$, one-way ANOVA with Tukey's post hoc) and $\mathbf{f}$ the cumulative probability distributions (GluN1 vs. GluA1 ${ }^{\star \star} p=0.0023$, GluN1 vs. GluA2 ${ }^{\star \star \star} p<0.0001$, GluA1 vs. GluA2 $p=0.3623$, two-tailed K-S test) of center-tocenter distances between PSD-95 and co-localized GluN1 (red, $n=1463$ clusters), GluA1 (green, $n=755$ clusters) and GluA2 (yellow, $n=1457$ clusters) nanoclusters. $\mathbf{g}$ Model of the sub-synaptic nanoscale localization of synaptic (PSD-95 colocalized) NMDARs and AMPARs. Measurements in e and $\mathbf{f}$ were performed on a per cluster basis (see "Methods"). Bars represent the mean \pm SEM.

PSD proteins $20,25,36,41,42,44$. Alternatively, calcium sensors might be organized independently of the glutamate receptors with release sites stochastically distributed within the active zone.

To explore these possibilities, we measured the distance between the centers of Bassoon nanomodules and the centers of colocalized SYT1 nanomodules (Fig. 9b). In order to identify synapses, only Bassoon nanomodules that were juxtaposed to PSD-95 nanomodules were selected. At synapses, the center-tocenter distances between 3D-segmented SYT1 and Bassoon nanoclusters $(185 \mathrm{~nm} \pm 2.0 \mathrm{~nm})$ closely matched the AMPARs -PSD-95 center-to-center distances (GluA1 $=189 \pm 3.0 \mathrm{~nm}$, GluA2 $=186 \pm 2.1 \mathrm{~nm}$ ). Thus, similar to AMPAR localization at the PSDs, SYT1 is localized toward the edges of Bassoon marked active zones.

Modeling suggests that to optimize synaptic transmission, AMPARs might be located closer to the fast SYT1 calcium sensor than the NMDARs are 21,49 . The localization of SYT1 relative to PSD-95 and the position of NMDARs and AMPARs within 
a
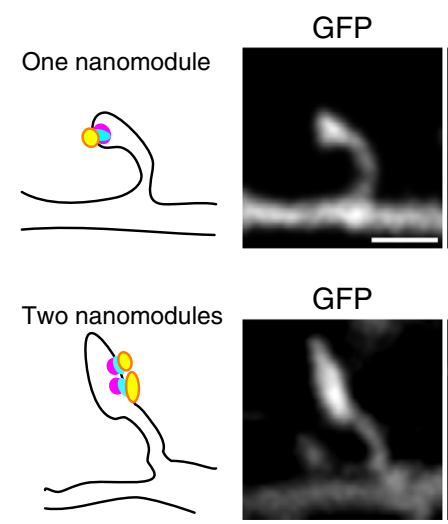

b

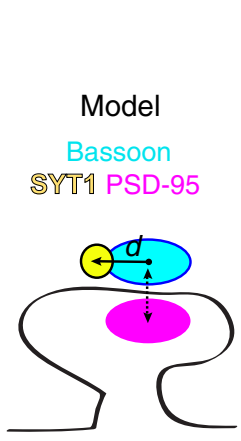

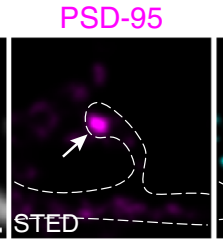

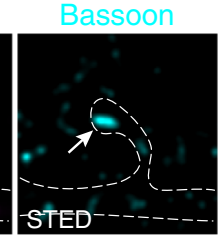

PSD-95
C

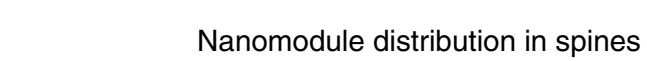

Nanomodule distribution in spines
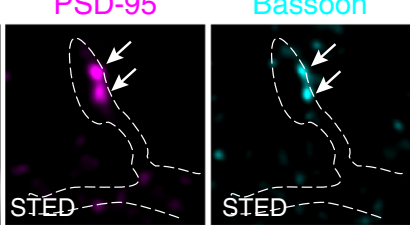

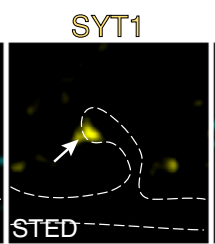

STED

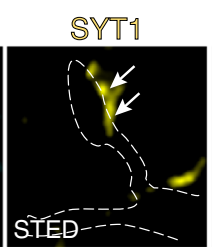

PSD-95

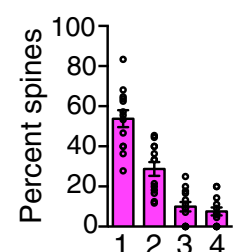

Number
Bassoon

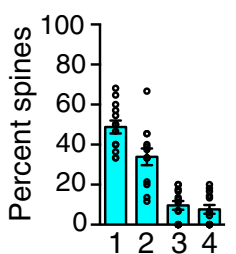

$\begin{array}{cc}1234 & 1 \\ 12 & 4\end{array}$

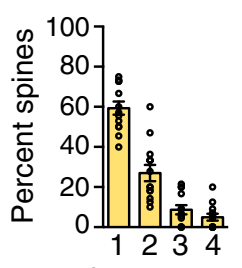

SYT1

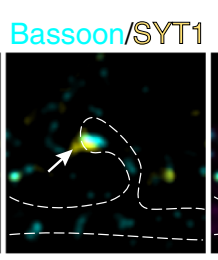

Three STED

channel merge

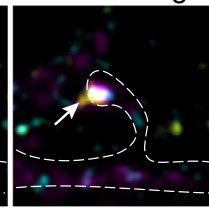

Three STED

Bassoon/SYT1 channel merge
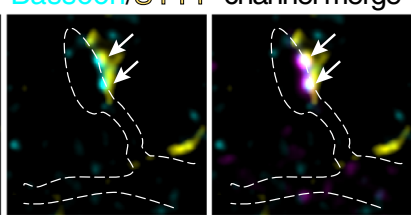

d

Nanomodule number relationship to spine size PSD-95 Bass. SYT1

Fig. 9 Nanoscale organization of SYT1 in dendritic spine synapses reflects the modular organization of pre- and postsynaptic adaptor molecules.

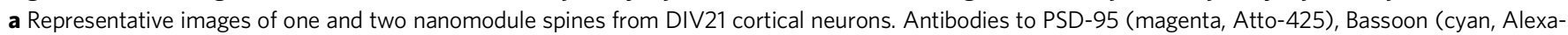

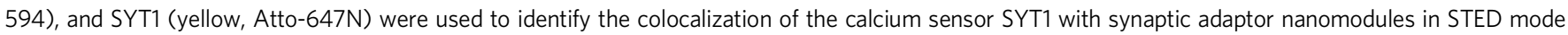
(arrows) in GFP-labeled spines (gray, dotted lines, confocal mode). Scale bar: $1 \mu \mathrm{m}$. b Schematic of the measurements performed to determine the organization of SYT1 relative to PSD-95-juxtaposed Bassoon nanomodules. c Percentage of spines containing single and multiple PSD-95, Bassoon, and SYT1 clusters ( $n=170$ spines, only spines with SYT1 were counted). d Quantification of the relationship between spine size ( $n=175$ spines) and the number of Bassoon (cyan line, $R^{2}=0.4449$, slope $=1.965 \pm 0.1669$ ), PSD-95 (magenta line, $R^{2}=0.4463$, slope $=1.892 \pm 0.1602$ ) and SYT1 (yellow line, $R^{2}=0.3369$, slope $=1.658 \pm 0.1768$ ) nanomodules ( $p=0.2068$, Bassoon vs. SYT1; $p=0.3268$, PSD-95 vs. Bassoon, one-way ANCOVA). Bars represent the mean \pm SEM.

individual PSD-95 nanomodules was determined in GFPtransfected DIV21-25 neurons labeled with antibodies against endogenous PSD-95, SYT1, and either GluN1 or GluA2 subunits of NMDARs and AMPARs (Fig. 10a). The distances between SYT1 and synaptic NMDARs, AMPARs, or PSD-95 were determined using nearest neighbor segmentation of STEDresolved nanoclusters of GluN1/PSD-95 colocalized clusters or GluA2/PSD-95 colocalized clusters and the centers of SYT1 clusters $^{46}$ (Fig. 10a). SYT1 clusters were significantly further from the centers of synaptic GluN1 clusters $(349 \pm 8.0 \mathrm{~nm})$ than from synaptic GluA2 clusters $(306 \pm 4.2 \mathrm{~nm}, p<0.0001$, two-tailed K-S test). On average, SYT1 clusters were $\sim 40 \mathrm{~nm}$ further from NMDARs than AMPARs (Fig. 10c). Thus, the distribution of the NMDARs shows that the bulk of NMDARs is located further from the SYT1 calcium sensor, but lie close to PSD-95 centers, while AMPARs at the PSD-95 periphery appear to be organized closer to SYT1 nanomodules (Fig. 10d). Consistent with this notion, center-to-center distances between SYT1 and PSD-95 nanomodules (either NMDAR or AMPAR colocalized) closely match the distances measured for GluN1 and SYT1 (GluA2 colocalized PSD-95: $347 \pm 6.3 \mathrm{~nm}, p=0.9967$; GluN1 colocalized PSD-95: $355 \pm 9.2 \mathrm{~nm}, p=0.9475)$, but are significantly longer when compared to GluA2 and SYT1 measurements (Fig. 10c, $p<0.0001$, one-way ANOVA).

The organization of SYT1 release sites in presynaptic terminals is closely linked to the localization of AMPARs in PSD-95 nanomodules (Fig. 10a-d). Three-dimensional reconstruction of GluA1, GluN1, and SYT1 clusters confirmed a closer apposition between SYT1 and AMPARs than between SYT1 and NMDARs (Supplementary Fig. $11 \mathrm{a}-\mathrm{c}$ ). Consistent with the above findings, the centers of SYT1 clusters were, on average, $35 \mathrm{~nm}$ closer to GluA1 clusters $(387 \pm 1.4 \mathrm{~nm})$ than GluN1-containing nanoclusters (Supplementary Fig. 11d, $422 \pm 2.1 \mathrm{~nm}, p<0.0001$, two-tailed Student's t-test). The distribution of center-to-center distances between SYT1 and GluA1 or GluN1 were similar to our findings with PSD-95-colocalized GluA2 and GluN1 subunits, supporting the validity of this approach (Supplementary Fig. 11e, $p<0.0001$, two-tailed $\mathrm{K}-\mathrm{S}$ test). These data indicate that synaptic AMPARs are on average closer to SYT1 nanoclusters than are synaptic NMDARs. Overall, our findings demonstrate that synchronous release sites segregate to sub-diffraction zones, mirroring the AMPARs' organization in spines. These data indicate the presence of specialized trans-synaptic nanomodules likely designed for a particular synaptic function (Fig. 10e).

\section{Discussion}

It has long been proposed that nanoscale precision in synaptic organization allows for the maximal efficiency of synaptic transmission and plasticity ${ }^{20}$. Here we define the subunit-specific rules that guide the organization of AMPAR and NMDAR heterotetramers and relate the organization of these proteins to both spine size and presynaptic calcium sensors. Both AMPAR and 
a
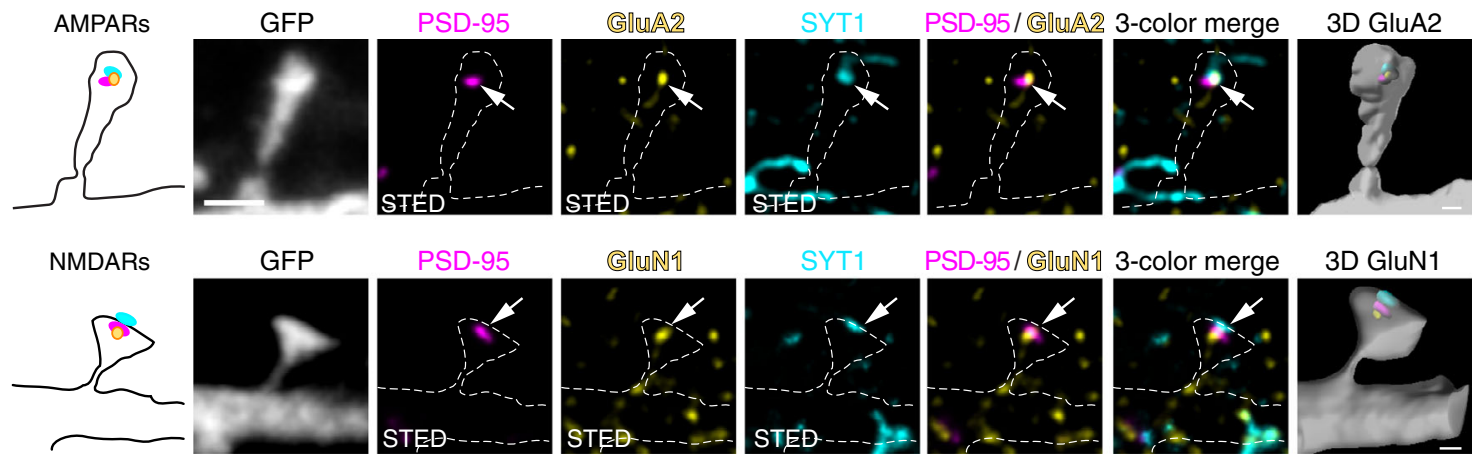

b

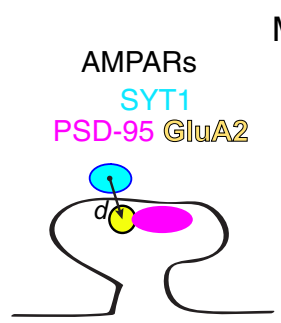

Model

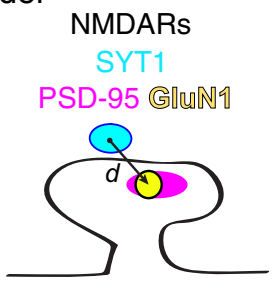

C

Center SYT1 to center GluRs SYT1 $\longrightarrow$ GluRs

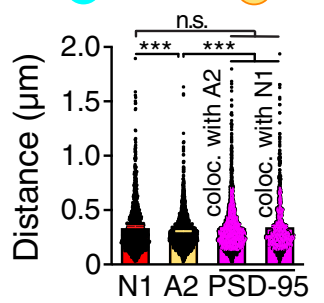

d

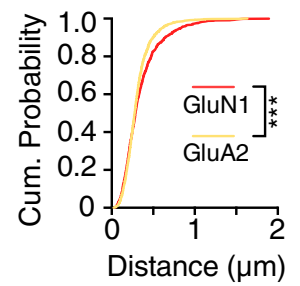

e
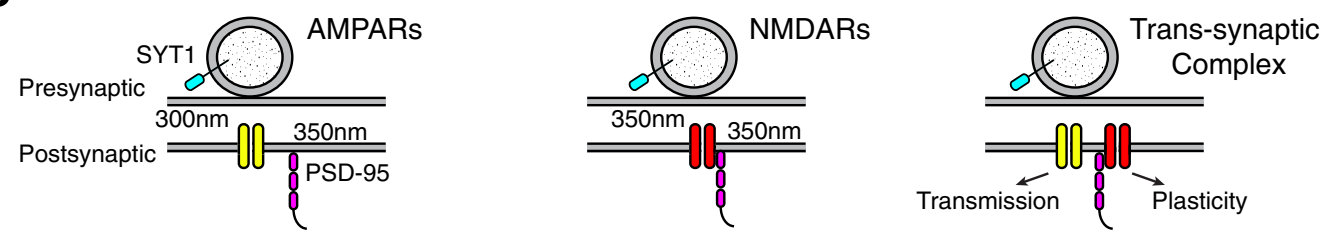

Fig. 10 NMDARs and AMPARs are distributed to distinct trans-synaptic nano-domains. a Representative images of one nanomodule spines from DIV21 cortical neurons immunostained for endogenous PSD-95 (magenta, Atto-425), SYT1 (cyan, AlexaFluor-594), and either AMPARs (GluA2, yellow, Atto$647 \mathrm{~N}$, top) or NMDARs (GluN1, yellow, Atto-647N bottom). Arrows show the juxtaposition between the SYT1 and synaptic (colocalized with PSD-95) AMPAR and NMDAR nanomodules in STED mode in the GFP-labeled spines (gray, dotted lines, confocal mode). Scale bar: $1 \mu \mathrm{m}$. b Schematic of the measurements of the center-to-center distances between SYT1 and synaptic (PSD-95 colocalized) NMDARs (GluN1) and AMPARs (GluA2). c Averages $\left({ }^{\star \star *} p<0.0001\right.$, one-way ANOVA with Tukey's post hoc) and $\mathbf{d}$ the cumulative probability distributions ( ${ }^{\star \star \star} p<0.0001$, two-tailed K-S test) of center-tocenter distances between SYT1 and synaptic (PSD-95-colocalized) GluN1 (red, $n=921$ clusters) or GluA2 (yellow, $n=1573$ clusters). Distances between the centers of GluA2 containing PSD-95 nanomodules ( $n=1340$ clusters) or GluN1 containing PSD-95 nanomodules ( $n=780$ clusters) and SYT1 are shown on the same graph. e Model of how the nanoscale sub-synaptic organization of the functional pre- and postsynaptic components of a synapse might be optimized for synaptic function. Measurements in $\mathbf{c}$ and $\mathbf{d}$ were performed on a per cluster basis (see "Methods"). Bars represent the mean \pm SEM.

NMDAR heterotetramers form modules that scale in number as spine size increases. The location of these modules is guided by function, with AMPARs closer to the fast calcium sensor SYT1 and NMDARs closer to nanomodules of PSD-95. These data support a model where AMPARs and NMDARs are positioned to maximize functional efficiency-with AMPARs located for optimal activation by glutamate and NMDARs located close to the scaffolding nexus to maximize their role in the induction of plasticity. Thus, despite their small size, single synapses likely contain specialized nanoscale sub-domains that allow for the optimization of synaptic function.

The organization of glutamate receptor heterotetramers is consistent with a nanoscale architecture that reflects the specific functions of glutamate receptor subunits and allows for flexibility likely needed for events such as synaptic plasticity. Both AMPA and NMDA receptors form nanomodules that scale with spine size, reminiscent of the modular organization of the MAGUK PSD-95 ${ }^{25,33}$. Increasing the number of glutamate receptor clusters in a modular fashion as spines increase in size would help maintain stable NMDAR-AMPAR ratios at individual synapses in response to ongoing plasticity ${ }^{50}$. These data are consistent with both the changes in AMPAR and NMDAR heterotetramer number that occur following structural plasticity $5,17,30$ and the stable AMPAR/NMDAR ratio seen after synaptic plasticity ${ }^{50-54}$ Within this modular framework there is flexibility generated by the specific subunit composition of AMPA and NMDA receptors.

AMPAR heterotetramers appear to be organized at the nanoscale for optimal subunit function, which generates a characteristic pattern of nanoscale organization of AMPAR subunits in relation to spine size. The pattern of AMPAR organization is reflected by differences in the organization of GluA1-containing AMPARs vs. those heterotetramers that lack GluA1. Nanoclusters of GluA2 subunit-containing AMPARs overall scaled in number as spine size increased and GluA2 immunostaining was localized to the majority $(89 \%)$ of spines. In contrast, GluA1-containing AMPARs ( $90 \%$ of which contain GluA2 subunits and are likely GluA1/A2 heterotetramers, (Supplementary Fig. 4d) 2,26,27 are primarily localized to large spines that contain two or more juxtaposed PSD-95/Bassoon nanomodules. These data are consistent with the model that AMPARs containing GluA1 subunits 
are found in larger spines and suggest that subunit composition is likely important for guiding their nanoscale localization at synapses $^{2}$.

The GluN2B subunit of the NMDAR is linked to the induction of synaptic plasticity and maturation or development of spine synapses $^{3}$. Consistent with this model, GluN2B subunitcontaining nanoclusters were enriched in smaller spines. This organization likely establishes a distinct class of NMDAR nanoclusters, as only GluN2B-containing nanoclusters did not exhibit positive scaling with spine size. Indeed, there is a negative relationship between the GluN2B nanocluster numbers and spine size. Given the role of these NMDAR subunits in tuning synaptic function and plasticity, the small, GluN2B-only containing spines are consistent with observations that spines with GluN2Bcontaining NMDAR heterotetramers might represent a group of spines that are primed for synaptic plasticity 7,37,55.

The rules governing NMDAR subunit distribution to larger spines appear straightforward and designed to allow for the flexible function of spine synapses. As spine size increases, GluN2A but not GluN2B-containing nanoclusters increase in number. These increases parallel increases in PSD-95 nanomodules and result in two types of larger spines: spines that contain GluN2A subunits or spines that contain a mixture of GluN2A and GluN2B subunit nanoclusters. These data are consistent with biochemical analyses showing a correlation between events that result in structural plasticity (LTP induction and sensory experience) and increases in levels of GluN2A-containing NMDAR density in the PSDs ${ }^{30,56,57}$. Defining the mechanisms that regulate the nanoscale organization of glutamate receptor heterotetramers in spines will be key for unraveling their functions at synapses.

Non-synaptic nanoclusters of AMPARs and NMDARs are abundant in spines but do not conform to the modular rules that drive the organization of synaptic nanoclusters of glutamate receptor heterotetramers. GluA2 subunit non-synaptic nanoclusters were found in most spines, while non-synaptic GluA1 and GluN1 subunit nanoclusters were found in only about $50 \%$ of spines. The abundance of these non-synaptic glutamate receptor nanoclusters is consistent with the presence of extrasynaptic, laterally diffusing glutamate receptors that may serve as a reservoir for delivery to and exchange with synaptic nanoclusters, particularly glutamate receptors containing GluA2 subunits ${ }^{18,19,58}$. This notion is further supported by our cLTP experiments in which we observed that NMDARdependent spine enlargement is linked to the formation of new GluA2 nanoclusters at synaptic sites, similar to what was previously reported for PSD-95 ${ }^{25}$. However, trafficking and nanorganization of GluA1 vs. GluA2 containing AMPARs to spines after plasticity is likely more complex especially given the findings that GluA1 and GluA2 subunits' synaptic localization is differentially controlled by their $\mathrm{N}$-terminal domains ${ }^{59}$. Non-synaptic nanoclusters also likely represent the pool of internalized and recycling glutamate receptors ${ }^{17}$. Consistent with these functions, non-synaptic nanoclusters of glutamate receptor heterotetramers were smaller than synaptic clusters ${ }^{13}$. The architecture of the spine, with clusters of non-synaptic and synaptic receptors that follow different sets of rules, highlights the importance of examining the relationship between neuronal morphology and the localization of pre- and postsynaptic proteins to understand the nanoscale organization of the synapse.

Exploration of the nanoscale organization of the synapse has revealed a complex and ordered network of pre- and postsynaptic proteins. Post-synaptic glutamate receptors and MAGUKs, and pre-synaptic Bassoon, vGlut1, SYT1, and Synaptophysin appear to share a modular organization where the number but not size of puncta of these proteins increase with increases in spine size $25,41,42,44$. Importantly most spines $(60 \%)$ have only one nanomodule, with few spines having more than two of these paired pre- and postsynaptic nanoclusters. Ultrastructural EM analysis of spines demonstrates the presence of spines with perforated postsynaptic densities and corresponding clusters of presynaptic features ${ }^{60-62}$. Moreover, changes in the number of perforated spines have been observed following the induction of structural plasticity in vivo ${ }^{63}$. Photostimulation induced structural plasticity at individual spines results in rapid increases in the number of perforated and segmented spines with a time course similar to changes in the nanoscale organization of synaptic proteins ${ }^{25,64}$. It will be of significant interest to determine the relationship between the nanoscale composition of synaptic proteins and the synaptic ultrastructure which will likely require the development of new methods including fixation protocols 65 .

While both NMDARs and AMPARs reflect the modular organization of PSD-95 in spines, they have distinct nanolocalizations within individual PSD-95 nanomodules. NMDAR nanoclusters are preferentially localized toward centers of PSD-95 nanomodules, whereas AMPAR nanoclusters are found at the periphery of PSD-95 nanomodules. These results support the model that NMDARs, but not AMPARs, are found at the core of PSDs ${ }^{13,39}$. This pattern of arrangement is also consistent with the differential stability of NMDARs and AMPARs at synapses. Lateral diffusion of AMPARs at synapses controls the number of AMPARs at PSDs and in plasticity ${ }^{19,58}$; therefore, their peripheral localization might enable the rapid exchange of AMPARs into and out of synapses. On the other hand, NMDARs appear to be much more stable at synapses, consistent with their interior nanoscale localization ${ }^{15}$. These data suggest that the nanoscale organization of the synapse is highly ordered.

Synaptic function is likely tightly linked to the nanoscale organization of AMPA and NMDA receptor sub-types ${ }^{13,20,41,42,66}$. Our data suggest that these proteins are highly ordered and localized in predictable ways. However, how such nanoscale patterning occurs remains unclear. Given that different subunits follow specific rules, it is likely interactions mediated directly by the receptor subunits themselves play key roles in the nanoscale patterning of these proteins. Attractive candidate mechanisms for establishing and regulating nanoscale synaptic organization include intracellular interactions ${ }^{3,17,45}$, extracellular interactions ${ }^{59,66-69}$, and liquidliquid phase separation ${ }^{70-72}$. How these mechanisms might establish functionally distinct synaptic sub-domains is currently an open question and that will require further investigation.

What is the importance of the distinct AMPAR and NMDAR nano-organization at synapses? Segregating AMPARs and NMDARs might allow for more flexibility in synaptic function. For example, the nanoscale localization of AMPARs at the periphery of individual PSD-95 nanomodules is tightly matched to the nanoscale organization of the fast SYT1 calcium sensor, essential for synchronous vesicle fusion, to the edges of Bassoon nanomodules. In contrast, NMDARs and SYT1 nanoclusters are organized somewhat independently of each other. This tight structural arrangement between synchronous sites of vesicle fusion and AMPARs is likely necessary for efficient AMPAR activation following the release of glutamate-due to their low affinity for glutamate $e^{1,21,49}$. The importance of such a precise nanoscale alignment between the sites of fusion and AMPARs was suggested by experiments in which the light-driven recruitment of AMPARs to synapses was insufficient to increase synaptic strength ${ }^{73}$. Consistent with our structural findings, the activation of AMPARs is much better coupled to the release of a quantum of glutamate than NMDARs ${ }^{13}$. The weaker coupling of NMDAR heterotetramers could be explained by a poorer alignment between NMDARs and SYT1. Alternatively, perhaps the 
differential localization of AMPAR and NMDAR heterotetramers reflects specialized nanoscale signaling hubs that contain relevant molecules required for specific synaptic functions and plasticity. Regardless, these data illuminate the robust rules of modularity that generate an exquisitely detailed organization of pre- and postsynaptic structures endowing synapses with the multifunctional but flexible nanoarchitecture necessary for neurotransmission and synaptic plasticity.

\section{Methods}

Animals. All animal studies were approved by the Institutional Animal Care and Use Committee guidelines at Thomas Jefferson University in accordance with the US National Institutes of Health guidelines. Long-Evans E17-18 male and female rat embryos from timed pregnant animals purchased from Charles River Laboratories Inc. (Wilmington, MA) were used to make primary cortical neuron cultures (see below).

Primary cortical neuron culture preparation. Dissociated cortical neurons were prepared from embryonic day 17-18 (E17-18) rat cerebral cortex as described previously ${ }^{25,74-76}$ and cultured in Neurobasal medium (cat\#: 21103049 , Thermo Fisher Scientific) supplemented with 1x B27 (cat\#: A3582801, Thermo Fisher Scientific), $2 \mathrm{mM}$ glutamine (cat\#: 25030081, Thermo Fisher Scientific) and penicillin-streptomycin (cat\#: 15140122, Thermo Fisher Scientific). Neurons were plated on poly-D-lysine (cat\#: 354210, Corning, Corning, NY) and laminin (cat\#: 354232, Corning, Corning, NY) coated glass coverslips (12 mm, \#1.5; Cat\#: 640712, Warner Instruments, Camden, CT). Neurons were plated at 150,000/well in 24-well plates and were maintained in a humidified $37^{\circ} \mathrm{C}$ incubator with $5 \% \mathrm{CO} 2$ until DIV21-25.

Neuronal transfection. Neurons were transfected at day in vitro 10 (DIV10) as previously described ${ }^{25}, 68,69,74,75$ using Lipofectamine 2000 (cat\#: 11668027, Thermo Fisher Scientific). EGFP under control of human ubiquitin promoter (pFUg-EGFP) was used as a cell filling dye to visualize neuronal morphology 25,74 . Briefly, the conditioned medium was first collected from plated neurons and replaced with $300 \mu \mathrm{l}$ of Neurobasal medium without any supplements (per one well of 24 -well plate). $100 \mu \mathrm{l}$ of transfection mix containing $0.5 \mu \mathrm{l}$ of Lipofectamine 2000 and $200 \mathrm{ng}$ of pFUg-EGFP plasmid was then added per well of a 24 -well plate. Neurons were incubated with the transfection cocktail at $37^{\circ} \mathrm{C}$ for $2 \mathrm{~h}$. After $2 \mathrm{~h}$, the transfection medium was replaced with $500 \mu \mathrm{l}$ of warmed conditioned medium, and neurons were placed in a humidified $37^{\circ} \mathrm{C}$ incubator until DIV21-25, at which point they were used for immunocytochemistry and STED.

Immunocytochemistry. For immunocytochemistry, cultured cortical neurons were fixed between DIV 21 and DIV25 in $4 \%$ paraformaldehyde (PFA)/2\% sucrose supplemented with $0.0025 \%$ glutaraldehyde (cat\#: 16000 , Electron Microscopy Sciences, Hatfield, PA) in PBS for $8 \mathrm{~min}$ at room temperature. Fixed neurons were then washed once in PBS, followed by a $15 \mathrm{~min}$ incubation at $4{ }^{\circ} \mathrm{C}$ in $1 \mathrm{mg} / \mathrm{ml}$ sodium borohydride solution (cat\#: 213462-25G, Millipore Sigma, St. Louis, MO) diluted in PBS. Coverslips were then washed three times in PBS, blocked, and permeabilized for $2 \mathrm{~h}$ at room temperature in $1 \%$ ovalbumin (cat\#: A5503, Millipore Sigma) and $0.2 \%$ gelatin from cold-water fish (cat\#: G7041-100G, Millipore Sigma) in PBS containing $0.01 \%$ saponin (cat\#: 47036, Millipore Sigma). Neurons were then stained for $2 \mathrm{~h}$ at room temperature or overnight at $4{ }^{\circ} \mathrm{C}$ with the indicated primary antibodies, washed three times in PBS, and then immunostained with corresponding secondary antibodies for $45 \mathrm{~min}$ at room temperature. After washing three times in PBS, coverslips were mounted with MOWIOL mounting medium and used for confocal and STED imaging 24 to $48 \mathrm{~h}$ post-mounting.

Antibodies. All primary and secondary antibodies were profiled in our previous publications and were reported to be specific ${ }^{25,68,69,74-77}$.

Primary antibodies used were: mouse monoclonal (IgG2A) anti-PSD-95 clone K28/43 (1:200, Neuromab, UC Davis, Davis, CA), mouse monoclonal IgG1 antiPSD-95 clone 7E3-1B8 (1:250, Thermo Fisher Scientific, Waltham, MA, cat\#: MA1-046) anti-guinea pig polyclonal anti-Bassoon (1:300, Synaptic Systems, Gottingen, Germany, cat\# 141 004), rabbit polyclonal anti-Bassoon (1:300, Synaptic Systems, cat \#: 141 003), chicken anti-GFP (1:2000, Abcam, Cambridge, MA, cat\# ab13970), rabbit monoclonal anti-GluN1 (1:500, AB9864, Millipore Sigma), mouse monoclonal (IgG2A) anti-GluN2A clone N327/95 (1:250, Neuromab), mouse monoclonal (IgG2B) anti-GluN2B clone N59/36 (1:250, Neuromab), mouse monoclonal (IgG1) anti-GluA1 clone N355/11 (1:250, Neuromab), rabbit polyclonal anti-GluA2(1:500, Synaptic Systems, cat\#: 182 103), mouse monoclonal (IgG2A) anti-Synaptotagmin 1 (1:500, Synaptic Systems, cat\#: 105 011).

Secondary antibodies used were: Goat anti mouse IgG2A Atto 425 (1:250, Rockland, Inc., cat\# 610-151-041), Goat anti-mouse IgG1 Atto-425 (1:250, Rockland, cat\#: 610-151-040), Goat anti-rabbit Atto 425 (1:250, Rockland, cat\#: 611-151-122), Goat anti-mouse IgG1 Atto-647N (1:500, Rockland, Inc., cat \# 610156-040), Goat anti-mouse IgG2A Atto-647N (1:500, Rockland, cat\#: 610-156
041), Goat anti mouse IgG2B Atto-647N (1:500, Rockland, cat\#: 610-156-042), Goat anti-rabbit Atto-647N (1:500, Rockland, Inc., cat \# 611-156-122), Goat antimouse IgG1 AlexaFluor-594 (1:500, Jackson ImmunoResearch, cat\# 115-587-185), Goat anti-mouse IgG2A AlexaFluor-594 (1:500, Jackson ImmunoResearch, cat\# 115-585-206), Donkey anti guinea pig AlexaFluor-594 (1:500, Jackson ImmunoResearch, cat \# 706-586-148). Donkey anti-Rabbit AlexaFluor-594 (1:500, Jackson ImmunoResearch cat \# 711-585-152).

Chemical LTP. NMDAR-dependent cLTP was induced by treatment of DIV 21-25, GFP-transfected neurons with glycine $(200 \mu \mathrm{M})$ as described ${ }^{25,28,29}$. Neurons were placed in artificial cerebrospinal fluid (ACSF, $143 \mathrm{mM} \mathrm{NaCl}, 5 \mathrm{mM} \mathrm{KCl}, 2 \mathrm{mM}$ $\mathrm{CaCl}_{2}, 1 \mathrm{mM} \mathrm{MgCl} 2,30 \mathrm{mM}$ glucose and $10 \mathrm{mM}$ HEPES, pH 7.4) containing $0.5 \mu \mathrm{M}$ TTX, $1 \mu \mathrm{M}$ strychnine and $20 \mu \mathrm{M}$ bicuculline. After imaging baseline morphology for 15-30 min at 6 min intervals, neurons were treated with $10 \mathrm{~mL}$ of glycine-stimulating solution $\left(143 \mathrm{mM} \mathrm{NaCl}, 5 \mathrm{mM} \mathrm{KCl}, 2 \mathrm{mM} \mathrm{CaCl}_{2}, 0 \mathrm{mM}\right.$ $\mathrm{MgCl}_{2}, 30 \mathrm{mM}$ glucose, $10 \mathrm{mM}$ HEPES, pH 7.4, $0.5 \mu \mathrm{M}$ TTX, $1 \mu \mathrm{M}$ strychnine, $20 \mu \mathrm{M}$ bicuculline and $200 \mu \mathrm{M}$ glycine) for $3-5 \mathrm{~min}$, followed by $10 \mathrm{~mL}$ of $0 \mathrm{mM}$ $\mathrm{MgCl}_{2}$ containing ACSF (143 mM NaCl, $5 \mathrm{mM} \mathrm{KCl}, 2 \mathrm{mM} \mathrm{CaCl}, 0 \mathrm{mM} \mathrm{MgCl}_{2}$, $30 \mathrm{mM}$ glucose, $10 \mathrm{mM}$ HEPES, $\mathrm{pH}$ 7.4, $0.5 \mu \mathrm{M}$ TTX, $1 \mu \mathrm{M}$ strychnine, and $20 \mu \mathrm{M}$ bicuculline). To block cLTP and spine enlargement, $50 \mu \mathrm{M}$ D-APV (D-2-amino-5 phosphonovalerate) and $10 \mu \mathrm{M}$ of MK-801 were included in the solutions described above. Control neurons were imaged in ACSF and not subjected to glycine. Neurons were imaged for $3 \mathrm{~h}$ at $6 \mathrm{~min}$ intervals to monitor long-term changes in spine size. Spines were classified as potentiated only if their area increased by $\geq 10 \%$ over baseline immediately following glycine treatment and remained enlarged throughout the entire imaging period.

Imaging-STED nanoscopy. Three-color STED imaging of endogenous synaptic proteins was performed as described previously ${ }^{25}$. Briefly, a Leica TCS SP8 gated STED (gSTED) 3X super-resolution system (Leica Microsystems) equipped with a tunable white light laser, CW 592 and $660 \mathrm{~nm}$ depletion lines, and a pulsed $775 \mathrm{~nm}$ depletion line was used for image acquisition. Resonance scanning $(8000 \mathrm{~Hz})$, gated HyD detectors (set at 100-200\%), and 100× oil immersion objective (Leica) with $5-10 \times$ zoom to obtain desired pixel size $(25 \mathrm{~nm})$ was used to acquire stacks at $150 \mathrm{~nm}$ image intervals. All data shown were imaged using 3X STED. Proteins labeled with Atto-647N or Alexa-594 secondary antibody conjugates were acquired using gSTED with HyD detectors adjusted between 0.2/0.3 to 6 ns. First, Atto-647N labeled endogenous proteins were excited with the $647 \mathrm{~nm}$ laser $(5-15 \%$ maximal laser power), and second, Alexa-594 labeled endogenous proteins were excited using the $594 \mathrm{~nm}$ laser (5-12\% maximal power). The pulsed $775 \mathrm{~nm}$ depletion line (set at $10-15 \%$ of maximal laser power for Atto-647N fluorophore and $35-50 \%$ of maximal laser power for Alexa-594 fluorophore) was used to generate STED with a resolution of $\sim 50 \mathrm{~nm}$, measured by determining the FWHM of puncta ${ }^{25}$ (Supplementary Fig. 2, see below). Lastly, proteins labeled with Atto-425 fluorophore were visualized by exciting with $442 \mathrm{~nm}$ line (12-15\% power), and the CW $592 \mathrm{~nm}$ line (50-65\% power) was used to generate STED. For this line, non-gated HyD detectors set to $250 \%$ gain were used to obtain super-resolved images $(\sim 80 \mathrm{~nm} \mathrm{XY}$ resolution, measured by the FWHM of puncta). For $Z$ depletion, 15\% of the 775 and $592 \mathrm{~nm}$ depletion line power was re-directed to the $Z$ donut to achieve an image $Z$-resolved at $\sim 250-300 \mathrm{~nm}^{25}$.

For resolution determination, single Alexa 594 fluorophores, identified as the smallest single puncta in the background, were imaged in $X Y$ in confocal and STED with $30 \% X Y 775 \mathrm{~nm}$ depletion either with $0 \% \mathrm{Z}$-Depletion power or $15 \% \mathrm{Z}$ Depletion power (Supplementary Fig. 2a). Full width at half maximum (FWHM) was measured from Gaussian fits of individual line plot profiles from lines drawn horizontally through puncta centers (white arrows, Supplementary Fig. 2a; individual line plot profiles: gray lines Supplementary Fig. 2b; average Gaussian fit: green lines Supplementary Fig. 2b). The point-to-point resolution was determined using GATTA $50 \mathrm{~nm}$ spaced Alexa 647 beads (GATTAQUANT GMBH, Grafelfing, Germany) imaged in confocal and STED with $775 \mathrm{~nm}$ depletion with $0 \% \mathrm{Z}$-depletion. Of note, the Alexa 647 dye is different than the dye used in immunolabeling (Atto $647 \mathrm{~N}$ ) and thus the resolution may vary slightly. The two GATTA beads spaced $50 \mathrm{~nm}$ apart were measured in STED with or without deconvolution (Supplementary Fig. 2d). Peak-to-peak distances were calculated from line plot profiles from lines drawn through the centers of both resolved beads (Supplementary Fig. 2d, e, raw data - gray traces, average - red line). To determine the dual-color STED point-to-point resolution, GATTA Dual color $70 \mathrm{~nm}$ Alexa 647/ Alexa-594/ Alexa 647 spaced beads were imaged in confocal and STED with $775 \mathrm{~nm}$ depletion laser with $0 \% \mathrm{Z}$-depletion (Supplementary Figure $2 \mathrm{~g}$, h). Peak-topeak distances were calculated from line plot profiles of the three beads by passing a line through all three peaks of the dual color GATTA beads (Supplementary Fig. 2g, h)

Time-lapse imaging of live neurons was performed with either the confocal spinning disk or Leica SP8. The confocal spinning disk was equipped with a Yokogawa CSU-10 and a Hamamatsu EM-CCD digital camera attached to an inverted Lecia microscope and controlled by Metamorph software 7.10. 2-4 $\mu \mathrm{m}$ image stacks were collected with an optical sectioning of $0.2 \mu \mathrm{m}$ using $100 \times$ (spinning disk), or $0.35 \mu \mathrm{m}$ using $63 \times$ (Leica SP8) oil immersion objectives. Adaptive focus control was utilized throughout the duration of time-lapse imaging 
Following imaging, neurons were immediately fixed, stained, and imaged using three-color STED as previously reported ${ }^{25}$.

Image processing and deconvolution. Detailed methodology for image processing, along with the FWHM measurements, is described in the second supplemental figure in Hruska et al. ${ }^{25}$. Briefly, images collected using the SP8 Leica gSTED from cultured neurons were deconvolved as stacks using Huygens deconvolution software (SVI, Hilversum, Netherlands) by specifying the point spread function (PSF, Leica SP8/DM6000/100 $\times$ objective, imaging wavelength), optical sectioning, $X, Y$, and $Z$ pixel resolution ${ }^{78}$. Deconvolution was performed separately for each channel using a maximum of 40 iterations. Each image was deconvolved using the same parameters. The effect of deconvolution on nanocluster sizes is minimal since the clusters sizes' are larger than the reported resolution.

Image analysis. Image analysis was conducted off-line using Fiji Image J (https:// imagej.net/Fiji) and built-in macros, as described below.

For nanomodule identification, super-resolution analysis of synaptic cluster localization in dendritic spines was performed on a per spine basis. Images of spines, acquired at confocal resolution $(\sim 250-300 \mathrm{~nm})$, were detected visually, and Gaussian blur (2-pixel value) was applied to filter out noise. Individual spines were converted to binary masks by thresholding the resulting EGFP image. Spine edges were determined by thresholding EGFP to the mean $+2 \times \mathrm{SD}$ (Standard Deviation) of the $1024 \times 1024$ pixel area corresponding to the entire image field. Nanoclusters of synaptic proteins (acquired in STED super-resolution) were identified by binarizing each channel separately using intensity thresholds. Thresholds were defined as the mean $+2 \times$ SD of intensity values of a $50 \times 50$ image pixel area. For three-color STED using gated detectors, clusters were defined as a minimum of 10 and a maximum of 100 continuous pixels corresponding to an area of $0.002-0.15 \mu \mathrm{m}^{2}$. The separation between neighboring STED resolved clusters were identified from the line intensity profiles of nearby clusters and was defined as the mean $+1.5 \times \mathrm{SD}$ of a local $50 \times 50$ pixel area that approximately corresponded to the maximum size of a spine head. The resulting thresholded nanomodules were used to determine whether these modules colocalized with individual spines. Only spines with clearly identifiable PSD-95 and/or Bassoon clusters were included in the analysis.

For 3D STED cluster assignments, outlines of spines were determined in individual $\mathrm{Z}$ sections of thresholded images. ROIs (region of interest) of each thresholded spine head were used to manually assign STED-resolved puncta to spines. PSD-95, AMPARs, and NMDARs clusters were assigned to a spine if the thresholded pixel areas were entirely within the spine head ROI. Bassoon and SYT1 clusters were assigned to a spine if the thresholded pixel areas either entirely or partially overlapped with the spine head ROI. Spine ROI colocalization of each cluster was made independently for each $Z$ section. Orthogonal views of the overlaid image stacks were used to verify that individual clusters colocalized with the spine ROI in the $Z$ plane. Finally, image stacks were overlaid and filtered by an edge-preserving algorithm in Imaris software (Bitplane AG 8.3.1). High-contrast images of puncta within the area that corresponded to the size of the spine head and shaft (approximately $100 \times 100$ pixels) were projected in Imaris to generate high-contrast volume rendered images. Volume rendering was performed for each channel separately using a two-voxel separation between thresholded objects. Thresholded clusters that did not colocalize with the area of the spine were discarded. Data for spine analysis of module number represent observations and were acquired and analyzed with an experimenter blinded to the condition during analysis.

Colocalization and nearest neighbor analysis of 3D STED-resolved synaptic clusters were performed on a per-cluster basis using the entire area of an image $(1024 \times 1024$ pixel format). Segmentation and subsequent measurements of distances of segmented clusters were performed using the DiAna plugin in Image J that enabled the analysis to be done in an automated way ${ }^{46}$. Segmentation of all synaptic clusters was performed using the local maxima method combined with user-defined thresholds ${ }^{46}$. Local maxima in $3 \mathrm{D}$ were identified using a radius of 3 pixels in the $X Y$ plane and 2 pixels in the $Z$ plane. Since deconvolved images were used for this analysis, the noise was set to zero. Local thresholds were determined by thresholding individual 16-bit images $(1024 \times 1024$ pixel format $)$ in each channel and were determined to be between 10,000 and 20,000 arbitrary units (AU). The maximum radius of segmented clusters was set to 8 pixels (individual pixel sizes in our images were set to $23-25 \mathrm{~nm}$ to allow maximum resolution of $\sim 50 \mathrm{~nm}$ ). The standard deviation for Gaussian fit and threshold calculation was set to 1.5 . Minimum and maximum voxel sizes were set to 3 and 20,000, respectively. Distance analysis of segmented clusters is based on classical Euclidean distance computation ${ }^{46}$. For trans-synaptic cluster measurements, we implemented centerto-center distances where, for each object from one image (channel 1), the centerto-center distances with all objects from another image (channel 2) were computed in 3D, and closest neighbor distances were reported. For these measurements, we only included objects that had clear juxtaposition between pre- and post-synaptic markers. For post-synaptic only measurements (PSD-95 with either AMPARs or NMDARs) or pre-synaptic only measurements (Bassoon with SYT1), we calculated distances only for colocalized objects in order to determine the positioning of synaptic AMPARs or NMDARs, and SYT1. For GluN2A-GluN2B distance measurements (Supplementary Fig. 9e-g), we calculated distances only between
GluN2A and GluN2B clusters that were both colocalized with a PSD-95 nanomodule within spine heads.

For chromatic aberration analysis, neurons transfected with GFP were imaged at DIV 21-25 and stained for endogenous Bassoon with three different antibodies against the same primary antibody (Rb Atto-425, Rb AlexaFluor-594, Rb Atto$647 \mathrm{~N}$ ) (Supplementary Fig. 1a). Triple-colocalized puncta were selected for analysis based on whether they had each of the three colors at similar brightness. The peakto-peak distances between each secondary antibody was determined from line plot profiles of each of three secondaries, measured by averaging the line plot profiles of four lines passing through the center of the triple-colocalized puncta for each of the three secondaries (Supplementary Fig. 1a, indicated by the white dashed line). In the axial plane, peak-to-peak distances were determined from the line plot profiles measured by passing a line through the center of each triple-labeled Bassoon puncta along the Z-plane (Supplementary Fig. 1d, dashed black line).

Statistics and reproducibility. Data were acquired and analyzed based on the standards in the field; however, no method of randomization was used to determine how samples were allocated to experimental groups and processed. Unless otherwise stated, data in figures and text are expressed as means \pm SEM. All data points collected were included for analysis. Statistics were performed using GraphPad Prism 8.0. Statistical significance of the differences among groups was determined by one-way analysis of variance followed by post hoc tests as described in individual figure legends, or by two-tailed Student's t-test when testing differences between two conditions. Kolmogorov-Smirnov (K-S) tests were used to test differences between non-parametric probability distributions. $P$ values less than 0.05 were considered statistically significant. For $p$ values less than 0.0001 or greater than 0.9999 , we are providing a range and not the exact number. The data distribution was assumed to be normal, but this was not formally tested. Sample sizes were determined based on our previous publication ${ }^{25,68,69,74-77}$. We also performed power analysis using the power of $0.8-0.95$ for medium and large effect sizes (as found in our preliminary studies) in G power software 3.1 to validate our sample sizes for statistical analyses. Group differences in variance were tested for each data set and determined to be similar. Unless stated otherwise, statistical tests were conducted on a per spine basis. Statistical tests for center-to-center distances between 3D STED clusters were performed on a per cluster basis. Data were collected from a minimum of nine different neurons acquired from three independent transfection experiments unless otherwise stated in figure legends.

Reporting summary. Further information on research design is available in the Nature Research Reporting Summary linked to this article.

\section{Data availability}

Data supporting the findings of this study are available within the article, its Supplementary Information and Source Data files. Additional information and relevant data will be available from the corresponding author upon reasonable request. The Source Data underlying Figs. 1b-i, 2e-i, 3b-h, 4b-f, 5b-h, 6b-g, 7b-d, 8e, f, 9c, d, 10c, d and Supplementary Figs. 1b, c, e, f, 2b, c, e, f, h-k, 4b-f, 6a, b, 9c-j, 10c, d, 11d, e are provided with this paper. Source data are provided with this paper.

Received: 19 January 2021; Accepted: 20 January 2022; Published online: 17 February 2022

\section{References}

1. Traynelis, S. F. et al. Glutamate receptor ion channels: Structure, regulation, and function. Pharmacol. Rev. 62, 405-496 (2010).

2. Diering, G. H. \& Huganir, R. L. The AMPA receptor code of synaptic plasticity. Neuron 100, 314-329 (2018).

3. Lau, C. G. \& Zukin, R. S. NMDA receptor trafficking in synaptic plasticity and neuropsychiatric disorders. Nat. Rev. Neurosci. 8, 413-426 (2007).

4. Paoletti, P., Bellone, C. \& Zhou, Q. NMDA receptor subunit diversity: Impact on receptor properties, synaptic plasticity, and disease. Nat. Rev. Neurosci. $\mathbf{1 4}$ 383-400 (2013)

5. Hayashi, Y. et al. Driving AMPA receptors into synapses by LTP and CaMKII: requirement for GluR1 and PDZ domain interaction. Science 287, 2262-2267 (2000).

6. Shi, S.-H., Hayashi, Y., Esteban, J. A. \& Malinow, R. Subunit-specific rules governing AMPA receptor trafficking to synapses in hippocampal pyramidal neurons. Cell 105, 331-343 (2001)

7. Liao, D., Hessler, N. A. \& Malinow, R. Activation of postsynaptically silent synapses during pairing-induced LTP in CA1 region of hippocampal slice. Nature 375, 400-404 (1995).

8. Petralia, R. S. et al. Selective acquisition of AMPA receptors over postnatal development suggests a molecular basis for silent synapses. Nat. Neurosci. 2, 31-36 (1999) 
9. Barria, A. \& Malinow, R. Subunit-specific NMDA receptor trafficking to synapses. Neuron 35, 345-353 (2002).

10. Bellone, C. \& Nicoll, R. A. Rapid bidirectional switching of synaptic NMDA receptors. Neuron 55, 779-785 (2007).

11. Kopec, C. D., Real, E., Kessels, H. W. \& Malinow, R. GluR1 links structural and functional plasticity at excitatory synapses. J. Neurosci. 27, 13706-13718 (2007).

12. Tanaka, H. \& Hirano, T. Visualization of subunit-specific delivery of glutamate receptors to postsynaptic membrane during hippocampal long-term potentiation. Cell Rep. 1, 291-298 (2012).

13. Goncalves, J. et al. Nanoscale co-organization and coactivation of AMPAR, NMDAR, and mGluR at excitatory synapses. Proc. Natl Acad. Sci. https:// doi.org/10.1073/pnas.1922563117 (2020).

14. Chater, T. E. \& Goda, Y. The role of AMPA receptors in postsynaptic mechanisms of synaptic plasticity. Front. Cell Neurosci. 8, 401 (2014).

15. Groc, L. et al. Differential activity-dependent regulation of the lateral mobilities of AMPA and NMDA receptors. Nat. Neurosci. 7, 695-696 (2004).

16. Groc, L. et al. NMDA receptor surface mobility depends on NR2A-2B subunits. Proc. Natl Acad. Sci. USA 103, 18769-18774 (2006).

17. Huganir, R. L. \& Nicoll, R. A. AMPARs and synaptic plasticity: The last 25 years. Neuron 80, 704-717 (2013).

18. Opazo, P., Sainlos, M. \& Choquet, D. Regulation of AMPA receptor surface diffusion by PSD-95 slots. Curr. Opin. Neurobiol. 22, 453-460 (2012).

19. Penn, A. C. et al. Hippocampal LTP and contextual learning require surface diffusion of AMPA receptors. Nature 549, 384 (2017).

20. Biederer, T., Kaeser, P. S. \& Blanpied, T. A. Transcellular nanoalignment of synaptic function. Neuron 96, 680-696 (2017).

21. Raghavachari, S. \& Lisman, J. E. Properties of quantal transmission at CA1 synapses. J. Neurophysiol. 92, 2456-2467 (2004).

22. Hardingham, G. E. \& Bading, H. Synaptic versus extrasynaptic NMDA receptor signalling: Implications for neurodegenerative disorders. Nat. Rev. Neurosci. 11, 682-696 (2010).

23. Liu, L. et al. Role of NMDA receptor subtypes in governing the direction of hippocampal synaptic plasticity. Science 304, 1021-1024 (2004)

24. Massey, P. V. et al. Differential roles of NR2A and NR2B-containing NMDA receptors in cortical long-term potentiation and long-term depression. $J$. Neurosci. 24, 7821-7828 (2004).

25. Hruska, M., Henderson, N., Marchand, S. J., Jafri, H. \& Dalva, M. B. Synaptic nanomodules underlie the organization and plasticity of spine synapses. Nat. Neurosci. 21, 671-682 (2018).

26. Lu, W. et al. Subunit composition of synaptic AMPA receptors revealed by a single-cell genetic approach. Neuron 62, 254-268 (2009).

27. Zhao, Y., Chen, S., Swensen, A. C., Qian, W.-J. \& Gouaux, E. Architecture and subunit arrangement of native AMPA receptors elucidated by cryo-EM. Science https://doi.org/10.1126/science.aaw8250 (2019).

28. Lu, W. et al. Activation of synaptic NMDA receptors induces membrane insertion of new AMPA receptors and LTP in cultured hippocampal neurons. Neuron 29, 243-254 (2001).

29. Park, M., Penick, E. C., Edwards, J. G., Kauer, J. A. \& Ehlers, M. D. Recycling endosomes supply AMPA receptors for LTP. Science 305, 1972-1975 (2004).

30. Grosshans, D. R., Clayton, D. A., Coultrap, S. J. \& Browning, M. D. LTP leads to rapid surface expression of NMDA but not AMPA receptors in adult rat CA1. Nat. Neurosci. 5, 27-33 (2001).

31. Zhang, Y., Cudmore, R. H., Lin, D.-T. T., Linden, D. J. \& Huganir, R. L. Visualization of NMDA receptor-dependent AMPA receptor synaptic plasticity in vivo. Nat. Neurosci. 18, 402-407 (2015).

32. Roth, R. H. et al. Cortical synaptic AMPA receptor plasticity during motor learning. Neuron https://doi.org/10.1016/j.neuron.2019.12.005 (2019).

33. Kornau, H. C., Schenker, L. T., Kennedy, M. B. \& Seeburg, P. H. Domain interaction between NMDA receptor subunits and the postsynaptic density protein PSD-95. Science 269, 1737-1740 (1995).

34. Hestrin, S. Developmental regulation of NMDA receptor-mediated synaptic currents at a central synapse. Nature https://doi.org/10.1038/357686a0 (1992).

35. Tovar, K. R. \& Westbrook, G. L. The incorporation of NMDA receptors with a distinct subunit composition at nascent hippocampal synapses in vitro. $J$. Neurosci. 19, 4180-4188 (1999).

36. Kellermayer, B. et al. Differential nanoscale topography and functional role of GluN2-NMDA receptor subtypes at glutamatergic synapses. Neuron 100, 106-1190000000 (2018).

37. Barria, A. \& Malinow, R. NMDA receptor subunit composition controls synaptic plasticity by regulating binding to CaMKII. Neuron 48, 289-301 (2005).

38. Matta, J. A., Ashby, M. C., Sanz-Clemente, A., Roche, K. W. \& Isaac, J. mGluR5 and NMDA receptors drive the experience- and activity-dependent NMDA receptor NR2B to NR2A subunit switch. Neuron 70, 339-351 (2011).

39. Chen, X. et al. PSD-95 family MAGUKs are essential for anchoring AMPA and NMDA receptor complexes at the postsynaptic density. Proc. Natl Acad. Sci. USA 112, 92 (2015).
40. Elias, G. M. \& Nicoll, R. A. Synaptic trafficking of glutamate receptors by MAGUK scaffolding proteins. Trends Cell Biol. 17, 343-352 (2007).

41. MacGillavry, H. D., Song, Y., Raghavachari, S. \& Blanpied, T. A. Nanoscale scaffolding domains within the postsynaptic density concentrate synaptic AMPA receptors. Neuron 78, 615-622 (2013).

42. Nair, D. et al. Super-resolution imaging reveals that AMPA receptors inside synapses are dynamically organized in nanodomains regulated by PSD95. J. Neurosci. 33, 13204-13224 (2013).

43. Stein, V., House, D. R., Bredt, D. S. \& Nicoll, R. A. Postsynaptic density-95 mimics and occludes hippocampal long-term potentiation and enhances long term depression. J. Neurosci.: Off. J. Soc. Neurosci. 23, 5503-5506 (2003).

44. Tang, A.-H. H. et al. A trans-synaptic nanocolumn aligns neurotransmitter release to receptors. Nature 536, 210-214 (2016).

45. Won, S., Levy, J. M., Nicoll, R. A. \& Roche, K. W. MAGUKs: Multifaceted synaptic organizers. Curr. Opin. Neurobiol. 43, 94-101 (2017).

46. Gilles, J.-F., Santos, M., Boudier, T., Bolte, S. \& Heck, N. DiAna, an ImageJ tool for object-based 3D co-localization and distance analysis. Methods 115, 55-64 (2017).

47. Kaeser, P. S. \& Regehr, W. G. Molecular mechanisms for synchronous, asynchronous, and spontaneous neurotransmitter release. Physiology $\mathbf{7 6}$ 333-363 (2014)

48. Bacaj, T. et al. Synaptotagmin-1 and synaptotagmin-7 trigger synchronous and asynchronous phases of neurotransmitter release. Neuron 80, 947-959 (2013).

49. Savtchenko, L. P. \& Rusakov, D. A. Moderate AMPA receptor clustering on the nanoscale can efficiently potentiate synaptic current. Philos. Trans. R. Soc. B: Biol. Sci. 369, 20130167 (2014).

50. Watt, A. J., Sjöström, P. J., Häusser, M., Nelson, S. B. \& Turrigiano, G. G. A proportional but slower NMDA potentiation follows AMPA potentiation in LTP. Nat. Neurosci. 7, 518-524 (2004).

51. Groc, L., Gustafsson, B. \& Hanse, E. Spontaneous unitary synaptic activity in CA1 pyramidal neurons during early postnatal development: Constant contribution of AMPA and NMDA receptors. J. Neurosci. 22, 5552-5562 (2002).

52. Myme, C. I. O., Sugino, K., Turrigiano, G. G. \& Nelson, S. B. The NMDA-toAMPA ratio at synapses onto layer $2 / 3$ pyramidal neurons is conserved across prefrontal and visual cortices. J. Neurophysiol. 90, 771-779 (2003).

53. Umemiya, M., Senda, M. \& Murphy, T. H. Behaviour of NMDA and AMPA receptor-mediated miniature EPSCs at rat cortical neuron synapses identified by calcium imaging. J. Physiol. 521, 113-122 (1999).

54. Watt, A. J., Rossum, M. C. Wv, MacLeod, K. M., Nelson, S. B. \& Turrigiano, G. G. Activity coregulates quantal AMPA and NMDA currents at neocortical synapses. Neuron 26, 659-670 (2000).

55. Kirkwood, A., Lee, H.-K. \& Bear, M. F. Co-regulation of long-term potentiation and experience-dependent synaptic plasticity in visual cortex by age and experience. Nature 375, 328-331 (1995).

56. Baez, M. V., Cercato, M. C. \& Jerusalinsky, D. A. NMDA receptor subunits change after synaptic plasticity induction and learning and memory acquisition. Neural Plast. 2018, 5093048 (2018).

57. Grosshans, D. R., Clayton, D. A., Coultrap, S. J. \& Browning, M. D. Analysis of glutamate receptor surface expression in acute hippocampal slices. Sci. Signal 2002, pl8 (2002).

58. Campelo, T. et al. AMPAR-dependent synaptic plasticity initiates cortical remapping and adaptive behaviors during sensory experience. Cell Rep. 32, 108097 (2020).

59. Watson, J. F., Pinggera, A., Ho, H. \& Greger, I. H. AMPA receptor anchoring at CA1 synapses is determined by $\mathrm{N}$-terminal domain and TARP $\gamma 8$ interactions. Nat. Commun. 12, 5083 (2021).

60. Harris, K. M., Jensen, F. E. \& Tsao, B. Three-dimensional structure of dendritic spines and synapses in rat hippocampus (CA1) at postnatal day 15 and adult ages: Implications for the maturation of synaptic physiology and long-term potentiation. J. Neurosci.: Off. J. Soc. Neurosci. 12, 2685-2705 (1992).

61. Harris, K. M. \& Weinberg, R. J. Ultrastructure of synapses in the mammalian brain. Cold Spring Harbor Perspect. Biol. https://doi.org/10.1101/ cshperspect.a005587 (2012)

62. Toni, N., Buchs, P. A., Nikonenko, I., Bron, C. R. \& Muller, D. LTP promotes formation of multiple spine synapses between a single axon terminal and a dendrite. Nature https://doi.org/10.1038/46574 (1999).

63. Hongpaisan, J. \& Alkon, D. L. A structural basis for enhancement of longterm associative memory in single dendritic spines regulated by PKC. Proc. Natl Acad. Sci. USA 104, 19571-19576 (2007).

64. Sun, Y., Smirnov, M., Kamasawa, N. \& Yasuda, R. Rapid ultrastructural changes in the PSD and surrounding membrane after induction of structural LTP in single dendritic spines. J. Neurosci. 41, 7003-7014 (2021).

65. Zaharijević, A. et al. Book reviews. Aspasia 13, 188-240 (2019).

66. Ramsey, A. M. et al. Subsynaptic positioning of AMPARs by LRRTM2 controls synaptic strength. Sci. Adv. 7, eabf3126 (2021). 
67. Díaz-Alonso, J. et al. Subunit-specific role for the amino-terminal domain of AMPA receptors in synaptic targeting. Proc. Natl Acad. Sci. USA 114, 7136-7141 (2017). PMID - 28630296.

68. Hanamura, K. et al. Extracellular phosphorylation of a receptor tyrosine kinase controls synaptic localization of NMDA receptors and regulates pathological pain. PLoS Biol. 15, e2002457 (2017).

69. Washburn, H. R., Xia, N. L., Zhou, W., Mao, Y.-T. \& Dalva, M. B. Positive surface charge of GluN1 $\mathrm{N}$-terminus mediates the direct interaction with EphB2 and NMDAR mobility. Nat. Commun. 11, 570 (2020).

70. Zeng, M. et al. Reconstituted postsynaptic density as a molecular platform for understanding synapse formation and plasticity. Cell https://doi.org/10.1016/ j.cell.2018.06.047 (2018).

71. Zeng, M. et al. Phase separation-mediated TARP/MAGUK complex condensation and AMPA receptor synaptic transmission. Neuron https:// doi.org/10.1016/j.neuron.2019.08.001 (2019).

72. Zeng, M. et al. Phase transition in postsynaptic densities underlies formation of synaptic complexes and synaptic plasticity. Cell 166, 1163-1175.e1112 (2016).

73. Sinnen, B. L. et al. Optogenetic control of synaptic composition and function. Neuron 93, 646-660.e645 (2017).

74. Hruska, M., Henderson, N. T., Xia, N. L., Marchand, S. J. \& Dalva, M. B. Anchoring and synaptic stability of PSD-95 is driven by ephrin-B3. Nat. Neurosci. 18, 1594-1605 (2015).

75. Kayser, M. S., McClelland, A. C., Hughes, E. G. \& Dalva, M. B. Intracellular and trans-synaptic regulation of glutamatergic synaptogenesis by EphB receptors. J. Neurosci.: Off. J. Soc. Neurosci. 26, 12152-12164 (2006).

76. McClelland, A. C., Hruska, M., Coenen, A. J., Henkemeyer, M. \& Dalva, M. B. Trans-synaptic EphB2-ephrin-B3 interaction regulates excitatory synapse density by inhibition of postsynaptic MAPK signaling. Proc. Natl Acad. Sci. USA 107, 8830-8835 (2010).

77. Nolt, M. J. et al. EphB controls NMDA receptor function and synaptic targeting in a subunit-specific manner. J. Neurosci.: Off. J. Soc. Neurosci. 31, 5353-5364 (2011).

78. Schoonderwoert, V., Dijkstra, R., Luckinavicius, G., Kobler, O. \& Voort, H. V. D. Huygens STED deconvolution increases signal-to-noise and image resolution towards $22 \mathrm{~nm}$. Microsc. Today 21, 38-44 (2013).

\section{Acknowledgements}

We thank the members of the Dalva laboratory for critical reading of our manuscript and helpful suggestions and comments. We would like to kindly thank Dr. Mahmud Siddiqi of Carnegie Institution for Science/Embryology for allowing us to use their Leica SP8 3D gSTED system to collect important data. Grants from NIDA (DA022727), NINDS
(NS106906, NS111976, and NS110385), and Eagles Autism Challenge to M.B.D. and NIA (AG064630) to M.H. supported this work.

\section{Author contributions}

M.H. conceptualized the study, designed and conducted experiments, analyzed data, and wrote the paper, R.C. designed and conducted experiments, developed analysis tools, analyzed data, wrote the paper, and analyzed data, M.B.D. conceptualized the study, designed experiments, and wrote the paper.

\section{Competing interests}

These authors declare no competing interests.

\section{Additional information}

Supplementary information The online version contains supplementary material available at https://doi.org/10.1038/s41467-022-28504-4.

Correspondence and requests for materials should be addressed to Matthew B. Dalva.

Peer review information Nature Communications thanks Laurent Groc and the other, anonymous, reviewer(s) for their contribution to the peer review of this work.

Reprints and permission information is available at http://www.nature.com/reprints

Publisher's note Springer Nature remains neutral with regard to jurisdictional claims in published maps and institutional affiliations.

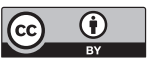

Open Access This article is licensed under a Creative Commons Attribution 4.0 International License, which permits use, sharing, adaptation, distribution and reproduction in any medium or format, as long as you give appropriate credit to the original author(s) and the source, provide a link to the Creative Commons license, and indicate if changes were made. The images or other third party material in this article are included in the article's Creative Commons license, unless indicated otherwise in a credit line to the material. If material is not included in the article's Creative Commons license and your intended use is not permitted by statutory regulation or exceeds the permitted use, you will need to obtain permission directly from the copyright holder. To view a copy of this license, visit http://creativecommons.org/ licenses/by/4.0/

(c) The Author(s) 2022 